\title{
Do It by Yourself: An Instructional Derivation of the Laplacian Operator in Spherical Polar Coordinates
}

\author{
Ana Laura Pérez-Martínez ${ }^{1}$, Maria del Pilar Aguilar-Del-Valle ${ }^{2}(\mathbb{C})$ and Arturo Rodríguez-Gomez ${ }^{2, *(\mathbb{D})}$ \\ 1 Facultad de Ingeniería, DCB, Universidad Nacional Autónoma de México, Ciudad de México 04510, Mexico; \\ ana.perez@ingenieria.unam.edu \\ 2 Instituto de Física, Universidad Nacional Autónoma de México, Circuito de la Investigación Científica s/n, \\ A.P. 20-364, Ciudad de México 04510, Mexico; pilarfc@ciencias.unam.mx \\ * Correspondence: arodriguez@fisica.unam.mx; Tel.: +52-55-5622-5000 (ext. 2618)
}

\section{check for}

updates

Citation: Pérez-Martínez, A.L.; Aguilar-Del-Valle, M.d.P.;

Rodríguez-Gomez, A. Do It by

Yourself: An Instructional Derivation

of the Laplacian Operator in

Spherical Polar Coordinates.

Mathematics 2021, 9, 2943. https://

doi.org/10.3390/math9222943

Academic Editors: David Pugalee,

Michelle Stephan and

Erdinç Çakıroğlu

Received: 18 October 2021

Accepted: 12 November 2021

Published: 18 November 2021

Publisher's Note: MDPI stays neutral with regard to jurisdictional claims in published maps and institutional affiliations.

Copyright: (c) 2021 by the authors. Licensee MDPI, Basel, Switzerland. This article is an open access article distributed under the terms and conditions of the Creative Commons Attribution (CC BY) license (https:/ / creativecommons.org/licenses/by/ $4.0 /)$.

\begin{abstract}
For scientists and engineers, the Laplacian operator is a fundamental tool that has made it possible to carry out important frontier studies involving wave propagation, potential theory, heat conduction, the distribution of stresses in a deformable solid and quantum mechanics. Knowing, understanding, and manipulating the Laplacian operator allows us to tackle complex and exciting physics, chemistry, and engineering problems. In this paper, contained in the Special Issue "Mathematics as the M in STEM Education", we present an instructional derivation of the Laplacian operator in spherical coordinates. Our derivation is self-contained and employs well-known mathematical concepts used in all science, technology, engineering, and mathematics (STEM) disciplines. Our lengthy but straightforward procedure shows that this fundamental tool in mathematics is not intractable but accessible to anyone who studies any of the STEM disciplines. We consider that this work may be helpful for students and teachers who wish to discuss the derivation of this vital tool from an elementary approach in their courses.
\end{abstract}

Keywords: Laplacian operator; spherical polar coordinates; undergraduate mathematics

\section{Introduction}

The acronym STEM stands for science, technology, engineering, and mathematics. Surprisingly, in STEM, math appears as a separate area and at the very end; this is possibly because, that way, the acronym is easier to remember and sounds better. However, it is not difficult to identify that, factually, mathematics is present in each discipline that makes up the STEM acronym. It is complicated to imagine a programming specialist who does not extensively use mathematics. The same is true for a physicist, a chemist, or any bachelor's degree in engineering.

The number of branches studied and investigated in mathematics is enormous and continues to grow. However, a good part of the mathematics belonging to science, technology, and engineering (STE) is literally used as a tool to solve specific problems. In this regard, there are mathematical tools that, due to their "generality", are present in all fields of STE, such as arithmetic, or basic algebra. Nevertheless, it is also possible to find "more sophisticated" mathematical tools that, in the same way, are present in all science, technology and engineering disciplines, such as in the case of the Laplacian operator.

The Laplacian operator is a differential operator that accepts one function and returns another. Usually, we find the Laplacian operator in partial differential equations. In fact, most of the time, we find it operating on the unknown function of the differential equation in question. It is precisely for this reason that its applications are wide and varied. Among these are fluid dynamics and heat conduction [1], electromagnetism [2,3], modern physics [4-7], condensed matter [8], general relativity [9], structure of matter [10] and quantum mechanics [11-14]. 
When one faces the task of solving a partial differential equation, the first thing to try is to propose a solution function composed of the multiplication of single-variable functions. When that is possible, the partial differential equation can be decomposed into a set of ordinary differential equations, which are more accessible to solve and whose solutions constitute the solution of the original partial differential equation. On many occasions, the above decomposition is NOT possible in a certain coordinate system, but it is possible in another. Therefore, it is advantageous to know the forms this differential operator (Laplacian) can take in different coordinate systems.

There are many spherical symmetries present in our universe; therefore, it is of particular interest to the science, technology, and engineering disciplines to know and understand the Laplacian operator's form in spherical polar coordinates. Its correct use has led to the generation of essential knowledge for humanity. For example, the quantum mechanical interpretation of the periodic table would not have been possible without the analytical solution of the Schrödinger equation for the hydrogen atom and the subsequent self-consistent calculations proposed by Hartree for multielectron atoms [15-17]. The Laplacian in spherical coordinates is also necessary for designing devices that involve beams of charged particles and in various electrostatic problems [18,19].

With the above examples, the reader might not be wholly convinced of the ubiquity of the Laplacian in STEM areas. However, talking purely about technology, it is imperative to mention that the Laplacian operator is a fundamental tool for image processing, which allows the quality of thousands of products worldwide to be controlled. In its discretized form in spherical coordinates, the Laplacian operator can process images from fisheye cameras [20], which would be very useful in video surveillance systems. The technological applications do not end there. In atmospheric sciences, the Laplacian operator in spherical coordinates is used to make models that allow us to determine how the shape of raindrops changes. It is important to know these oscillations in shape because they can influence the propagation of electromagnetic radiation used in telecommunications [21]. The list could go on; however, what is evident is that knowing, understanding, and manipulating the Laplacian operator provides scientists and engineers with a powerful tool that allows them to tackle interesting problems related to the STEM disciplines. In our experience as teachers, we have perceived that, although the tool is contained in multiple math software, graphing calculators, and a plethora of math formula handbooks, knowing the guts of its origin allows students to handle the operator with much more skill. Therefore, this paper presents an instructional and full-fledged derivation of the Laplacian operator in spherical polar coordinates starting from the Laplacian operator in rectangular coordinates. Although the subject has been extensively treated within the literature, here, we present it with an approach that attempts to reconcile the lack of detail with which most popular books on mathematics and physics address it [22,23], with the "sophistication" of shorter derivations that make use of non-trivial concepts such as the theory of complex variable [24], or the total angular momentum operator [25]. The strategy we follow was developed over several years of experience in teaching courses in mathematics, quantum physics, the structure of matter, electron microscopy, computer-aided design, and resistance of materials. Our self-contained procedure uses standard mathematical tools available from the second year of undergraduate studies. In addition, detailed explanations are implicitly included to attempt to solve doubts that recurrently appear during our courses. We believe that this work could be helpful for the self-taught student and for academics who wish to present it fully to students whose work involves using this important tool.

\section{Discussion}

We start by recalling the Nabla operator:

$$
\nabla=\frac{\partial}{\partial x} \hat{i}+\frac{\partial}{\partial y} \hat{j}+\frac{\partial}{\partial z} \hat{k}
$$


The above expression tells us that, if we apply the Nabla operator to a scalar function $\mathrm{f}$, we obtain a vector that indicates the direction of maximum increment of the function $\mathrm{f}$. Meanwhile, the Laplacian operator is the divergence of the gradient of a scalar function, that is,

$$
\nabla^{2}=\nabla \cdot \nabla
$$

If we apply the Laplacian to a scalar function, we have a scalar.

$$
\nabla^{2} \mathrm{f}(\mathrm{x}, \mathrm{y}, \mathrm{z})=\frac{\partial^{2} \mathrm{f}}{\partial \mathrm{x}^{2}}+\frac{\partial^{2} \mathrm{f}}{\partial \mathrm{y}^{2}}+\frac{\partial^{2} \mathrm{f}}{\partial \mathrm{z}^{2}}=\text { SCALAR }
$$

We should identify that, in this last expression, the unit vectors $\hat{i}, \hat{j}, \hat{k}$ no longer appear and the results of the three-second partial derivatives are added. Consequently, the Laplacian operator in spherical polar coordinates also has to give us a scalar quantity. Unfortunately, the Laplacian operator of a function that depends on " $r, \theta$ " and " $\phi$ " is NOT obtained by applying the definition of the Laplacian in rectangular coordinates.

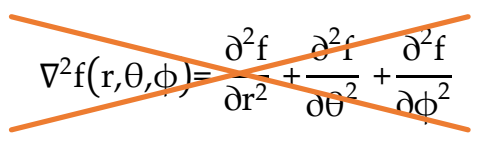

In order to derive the definition of the Laplacian operator in spherical polar coordinates, it is necessary to apply some simple rules of differential calculus with which every high school student is familiar.

In rectangular coordinates, we have that $\nabla^{2} f(x, y, z)=\frac{\partial^{2} f}{\partial x^{2}}+\frac{\partial^{2} f}{\partial y^{2}}+\frac{\partial^{2} f}{\partial z^{2}}$, when $f$ depends on $\mathrm{x}, \mathrm{y}, \mathrm{z}$; however, we should carry out a coordinate change when $\mathrm{f}$ depends on " $\mathrm{r}$," " $\theta$ " and " $\phi$ ", i.e., spherical polar coordinates. This change of coordinates can be treated as a variable change in the function we seek to derive. Let us recall the chain rule using Leibniz's notation:

$$
\frac{\mathrm{df}}{\mathrm{dx}}=\frac{\mathrm{df}}{\mathrm{du}} \frac{\mathrm{du}}{\mathrm{dx}}
$$

The above rule tells us that, if, initially, $\mathrm{f}$ is a function of $\mathrm{x}$ and we make a change in the variable from $\mathrm{x}$ to $\mathrm{u}$, then we have to derive $\frac{f(u)}{d u}$ and multiply by the derivative of $\mathrm{u}$ with respect to $\mathrm{x} \frac{d u}{d x}$.

When we change variables from $\mathrm{x}, \mathrm{y}, \mathrm{z}$ to $\mathrm{r}, \theta, \phi$, we have to identify that each rectangular variable is expressed using the three spherical variables. In other words, to describe the $x$ coordinate in spherical coordinates, one has to give a value for $r$, another for $\theta$ and another for $\phi$; the same occurs for $y$ and $z$ coordinates. Therefore, to find the derivative of $f(r, \theta, \phi)$ with respect to $\mathrm{x}$, we apply the chain rule three times. The same is true for $\mathrm{y}$ and for $\mathrm{z}$. Let us see that

$$
\begin{aligned}
& \frac{\partial f}{\partial x}=\frac{\partial f}{\partial r} \frac{\partial r}{\partial x}+\frac{\partial f}{\partial \theta} \frac{\partial \theta}{\partial x}+\frac{\partial f}{\partial \phi} \frac{\partial \phi}{\partial x} \\
& \frac{\partial f}{\partial y}=\frac{\partial f}{\partial r} \frac{\partial r}{\partial y}+\frac{\partial f}{\partial \theta} \frac{\partial \theta}{\partial y}+\frac{\partial f}{\partial \phi} \frac{\partial \phi}{\partial y} \\
& \frac{\partial f}{\partial z}=\frac{\partial f}{\partial r} \frac{\partial r}{\partial z}+\frac{\partial f}{\partial \theta} \frac{\partial \theta}{\partial z}+\frac{\partial f}{\partial \phi} \frac{\partial \phi}{\partial z}
\end{aligned}
$$

Let us identify that the derivatives of the "new" variables with respect to the "old" ones are nine quantities that we must calculate to obtain the Laplacian operator in spherical polar coordinates. Those derivatives are: $\frac{\partial r}{\partial x}, \frac{\partial \theta}{\partial x}, \frac{\partial \phi}{\partial x}, \frac{\partial r}{\partial y}, \frac{\partial \theta}{\partial y}, \frac{\partial \phi}{\partial y}, \frac{\partial r}{\partial z}, \frac{\partial \theta}{\partial z}$ and $\frac{\partial \phi}{\partial z}$. 
To carry out this task, we must first express $r, \theta, \phi$ in terms of $x, y, z$ and vice versa, i.e., to pose the coordinate transformations. Figure 1 shows a point $\mathrm{P}$ in a three-dimensional space where the coordinates $x, y, z$, and $r, \theta, \phi$ are indicated for the same point.

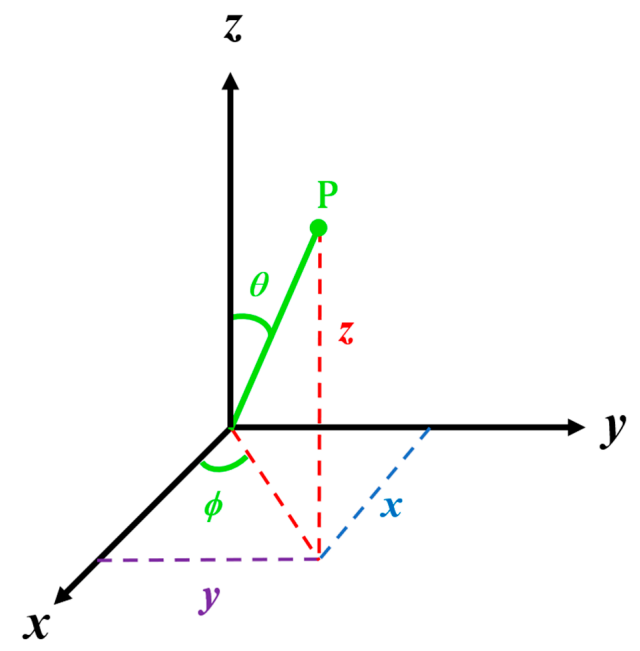

Figure 1. Spherical coordinates $r, \theta, \phi$ and their respective Cartesian coordinates $x, y, z$ for a point $P$ in a three-dimensional space.

We can relate the $x, y, z$ coordinates with $r, \theta, \phi$ using the different colored triangles that appear in Figure 1. Using the same color code, we can redraw, in 2D, the triangles of Figure 1, as indicated in Figures 2 and 3.

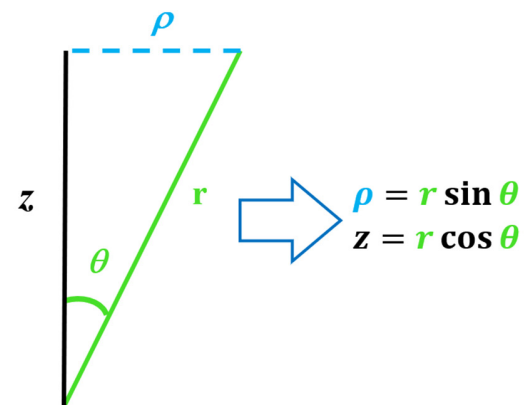

Figure 2. Triangles to express the rectangular coordinates $\mathrm{x}, \mathrm{y}, \mathrm{z}$ in terms of the spherical coordinates $\mathrm{r}, \theta, \phi$.

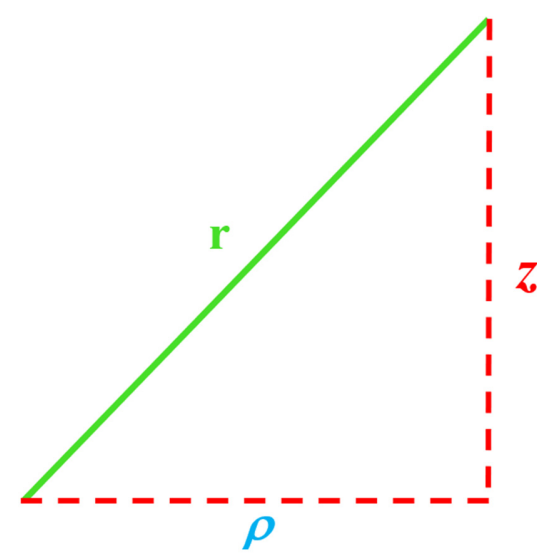

Figure 3. Triangle formed by the projection of $r$ in the $x-y$ plane. 
From Figure 2, it is easy to identify that the change of coordinates from $x, y, z$ to $r, \theta, \phi$ is as follows:

$$
\begin{gathered}
\mathrm{x}=\mathrm{r} \sin \theta \cos \phi \\
\mathrm{y}=\mathrm{r} \sin \theta \sin \phi \\
\mathrm{z}=\mathrm{r} \cos \theta
\end{gathered}
$$

To express $r, \theta, \phi$ in terms of $x, y, z$, we can also use Figures 2 and 3 . From Figure 2, it is possible to identify that $\rho^{2}=x^{2}+y^{2}$ and, from Figure 3 , one can observe that $\rho^{2}+z^{2}=r^{2}$; consequently, we can write

$$
\mathrm{r}^{2}=\mathrm{x}^{2}+\mathrm{y}^{2}+\mathrm{z}^{2}
$$

Using Figure 2, we can also write

$$
\cos \theta=\frac{\mathrm{z}}{\mathrm{r}} \rightarrow \theta=\cos ^{-1}\left(\frac{\mathrm{z}}{\left(\mathrm{x}^{2}+\mathrm{y}^{2}+\mathrm{z}^{2}\right)^{\frac{1}{2}}}\right)
$$

and

$$
\tan \phi=\frac{y}{x} \rightarrow \phi=\tan ^{-1}\left(\frac{y}{x}\right)
$$

We are now able to fully express the transformations from rectangular to spherical coordinates and vice versa.

$$
\begin{array}{cc}
\mathrm{x}=\mathrm{r} \sin \theta \cos \phi & \mathrm{r}=\sqrt{\mathrm{x}^{2}+\mathrm{y}^{2}+\mathrm{z}^{2}} \\
\mathrm{y}=\mathrm{r} \sin \theta \sin \phi & \theta=\cos ^{-1}\left(\frac{\mathrm{z}}{\left(\mathrm{x}^{2}+\mathrm{y}^{2}+\mathrm{z}^{2}\right)^{\frac{1}{2}}}\right) \\
\mathrm{z}=\mathrm{r} \cos \theta & \phi=\tan ^{-1}\left(\frac{\mathrm{y}}{\mathrm{x}}\right)
\end{array}
$$

With the coordinate transformations of Equation (2), we can calculate the nine derivatives of the "new variables" $(r, \theta, \phi)$ with respect to the "old variables" $(x, y, z)$. Let us start with $\frac{\partial r}{\partial x}$ :

$$
\frac{\partial r}{\partial x}=\frac{\partial}{\partial x}\left(\sqrt{x^{2}+y^{2}+z^{2}}\right)
$$

Let us make a change of variable as follows: $u=x^{2}+y^{2}+z^{2}$, then we have $\frac{\partial(\sqrt{u})}{\partial u} \frac{\partial u}{\partial x}$.

$$
\begin{gathered}
\frac{\partial(\sqrt{\mathrm{u}})}{\partial \mathrm{u}}=\frac{1}{2} \mathrm{u}^{-\frac{1}{2}}=\frac{1}{2^{\frac{1}{2}}}=\frac{1}{2 \sqrt{\mathrm{u}}} \\
\frac{\partial \mathrm{u}}{\partial \mathrm{x}}=\frac{\partial\left(\mathrm{x}^{2}+\mathrm{y}^{2}+\mathrm{z}^{2}\right)}{\partial \mathrm{x}}=2 \mathrm{x} \\
\frac{\partial(\sqrt{\mathrm{u}})}{\partial \mathrm{u}} \frac{\partial \mathrm{u}}{\partial \mathrm{x}}=\frac{1}{2 \sqrt{\mathrm{u}}}(2 \mathrm{x})=\frac{2 \mathrm{x}}{2 \sqrt{\mathrm{x}^{2}+\mathrm{y}^{2}+\mathrm{z}^{2}}}=\frac{\mathrm{x}}{\sqrt{\mathrm{x}^{2}+\mathrm{y}^{2}+\mathrm{z}^{2}}}
\end{gathered}
$$

Therefore,

$$
\frac{\partial \mathrm{r}}{\partial \mathrm{x}}=\frac{\mathrm{x}}{\mathrm{r}}
$$

By symmetry, we can anticipate that the calculations for $\frac{\partial r}{\partial y}$ and $\frac{\partial r}{\partial z}$ are exactly as the previous one; therefore,

$$
\frac{\partial \mathrm{r}}{\partial \mathrm{y}}=\frac{\mathrm{y}}{\mathrm{r}} ; \frac{\partial \mathrm{r}}{\partial \mathrm{z}}=\frac{\mathrm{z}}{\mathrm{r}}
$$


Let us continue with $\frac{\partial \theta}{\partial x}$.

$$
\frac{\partial \theta}{\partial x}=\frac{\partial}{\partial x}\left(\cos ^{-1}\left(\frac{\mathrm{z}}{\left(\mathrm{x}^{2}+\mathrm{y}^{2}+\mathrm{z}^{2}\right)^{\frac{1}{2}}}\right)\right)=\frac{\partial}{\partial \mathrm{x}}\left(\cos ^{-1}\left(\frac{z}{r}\right)\right)
$$

By implicit derivation, it is known that the derivative of the arc cosine is defined as follows:

$$
\frac{\mathrm{d}}{\mathrm{dx}}\left(\cos ^{-1}(f)\right)=-\frac{\frac{\mathrm{d}(f)}{\mathrm{dx}}}{\sqrt{1-f^{2}}}
$$

For our case, we have to perform the following:

$$
\begin{gathered}
\frac{\partial}{\partial x}\left(\cos ^{-1}\left(\frac{z}{r}\right)\right)=-\frac{\frac{\partial}{\partial x}\left(\frac{z}{r}\right)}{\sqrt{1-\left(\frac{z}{r}\right)^{2}}} \\
\frac{\partial}{\partial x}\left(\frac{z}{r}\right)=\frac{\partial}{\partial x}\left(\frac{\mathrm{z}}{\sqrt{\mathrm{x}^{2}+\mathrm{y}^{2}+\mathrm{z}^{2}}}\right)
\end{gathered}
$$

Let us make a change of variable as follows: $\mathrm{u}=\mathrm{x}^{2}+\mathrm{y}^{2}+\mathrm{z}^{2}$, hence we have to calculate $\frac{\partial\left(\frac{z}{\sqrt{u}}\right)}{\partial u} \frac{\partial u}{\partial x}$.

$$
\frac{\partial\left(\frac{\mathrm{z}}{\sqrt{\mathrm{u}}}\right)}{\partial \mathrm{u}}=\mathrm{z} \frac{\partial\left(u^{-\frac{1}{2}}\right)}{\partial \mathrm{u}}=-\frac{1}{2} \mathrm{z} \mathrm{u}^{-\frac{3}{2}}=-\frac{\mathrm{z}}{2 \mathrm{u}^{\frac{3}{2}}}
$$

We know that $u^{\frac{1}{2}}=r$; then,

$$
\begin{gathered}
\frac{\partial\left(\frac{z}{\sqrt{u}}\right)}{\partial u}=-\frac{z}{2\left(u^{\frac{1}{2}}\right)\left(u^{\frac{1}{2}}\right)\left(u^{\frac{1}{2}}\right)}=-\frac{z}{2 r^{3}} \\
\frac{\partial u}{\partial x}=\frac{\partial}{\partial x}\left(x^{2}+y^{2}+z^{2}\right)=2 x \\
\frac{\partial\left(\frac{z}{\sqrt{u}}\right)}{\partial u} \frac{\partial u}{\partial x}=-\frac{z}{2 r^{3}}(2 x)=-\frac{z x}{r^{3}}
\end{gathered}
$$

We insert this last result in the expression for the derivative of the arc cosine and we have

$$
\frac{\partial}{\partial x}\left(\cos ^{-1}\left(\frac{z}{r}\right)\right)=-\frac{\frac{\partial}{\partial x}\left(\frac{z}{r}\right)}{\sqrt{1-\left(\frac{z}{r}\right)^{2}}}=-\frac{\left(-\frac{z x}{r^{3}}\right)}{\sqrt{1-\left(\frac{z}{r}\right)^{2}}}
$$

Negative signs cancel out and it results

$$
\frac{\partial \theta}{\partial x}=\frac{\frac{z x}{r^{3}}}{\sqrt{1-\left(\frac{z}{r}\right)^{2}}}
$$

Remember that $z=r \cos \theta$ and $x=r \sin \theta \cos \phi$, while $\frac{z}{r}=\cos \theta$; thus,

$$
\frac{\partial \theta}{\partial x}=\frac{\frac{(r \cos \theta)(r \sin \theta \cos \phi)}{r^{3}}}{\sqrt{1-\cos ^{2} \theta}}
$$

Since $\sin ^{2} \theta+\cos ^{2} \theta=1$, then $\sin ^{2} \theta=1-\cos ^{2} \theta$ and $\sin \theta=\sqrt{1-\cos ^{2} \theta}$; therefore,

$$
\frac{\partial \theta}{\partial x}=\frac{\frac{(r \cos \theta)(r \sin \theta \cos \phi)}{r^{3}}}{\sin \theta}=\frac{\cos \theta \cos \phi}{r}
$$

Therefore,

$$
\frac{\partial \theta}{\partial x}=\frac{\cos \theta \cos \phi}{r}
$$


Let us continue with $\frac{\partial \theta}{\partial y}$.

$$
\frac{\partial \theta}{\partial y}=\frac{\partial}{\partial y}\left[\cos ^{-1}\left(\frac{\mathrm{z}}{\sqrt{\mathrm{x}^{2}+\mathrm{y}^{2}+\mathrm{z}^{2}}}\right)\right]=\frac{\partial}{\partial \mathrm{y}}\left[\cos ^{-1}\left(\frac{z}{r}\right)\right]
$$

Using the definition for the derivative of the arc cosine, we have

$$
\frac{\mathrm{d}}{\mathrm{dy}}\left(\cos ^{-1}(f)\right)=-\frac{\frac{\mathrm{d}(f)}{\mathrm{dy}}}{\sqrt{1-\mathrm{f}^{2}}}
$$

Applying it to our case,

$$
\frac{\partial}{\partial y}\left(\cos ^{-1}\left(\frac{z}{r}\right)\right)=-\frac{\frac{\partial}{\partial y}\left(\frac{z}{r}\right)}{\sqrt{1-\left(\frac{z}{r}\right)^{2}}}
$$

For $\frac{\partial}{\partial y}\left(\frac{z}{r}\right)$, we do not repeat the derivative because the procedure is exactly the same as that for the variable $\mathrm{x}$. We just have to substitute $\mathrm{x}$ for $\mathrm{y}$. Therefore,

$$
\frac{\partial}{\partial y}\left(\frac{z}{r}\right)=-\frac{z y}{r^{3}}
$$

Inserting this result into the expression for the derivative of the arc cosine, we have

$$
\frac{\partial}{\partial y}\left(\cos ^{-1}\left(\frac{z}{r}\right)\right)=-\frac{\frac{\partial}{\partial y}\left(\frac{z}{r}\right)}{\sqrt{1-\left(\frac{z}{r}\right)^{2}}}=\frac{\frac{z y}{r^{3}}}{\sqrt{1-\left(\frac{z}{r}\right)^{2}}}
$$

We know that $\mathrm{z}=\mathrm{r} \cos \theta ; \mathrm{y}=\mathrm{r} \sin \theta \sin \phi ; \frac{\mathrm{z}}{\mathrm{r}}=\cos \theta ; \sin \theta=\sqrt{1-\cos ^{2} \theta}$. The last expression we rewrite as follows:

$$
\frac{\partial \theta}{\partial y}=\frac{\frac{(r \cos \theta)(r \sin \theta \sin \phi)}{r^{3}}}{\sin \theta}=\frac{\cos \theta \sin \phi}{r}
$$

Whereby,

$$
\frac{\partial \theta}{\partial y}=\frac{\cos \theta \sin \phi}{r}
$$

Now, we carry out the corresponding procedure for $\frac{\partial \theta}{\partial z}$.

$$
\frac{\partial \theta}{\partial z}=\frac{\partial}{\partial z}\left[\cos ^{-1}\left(\frac{\mathrm{z}}{\sqrt{\mathrm{x}^{2}+\mathrm{y}^{2}+\mathrm{z}^{2}}}\right)\right]=\frac{\partial}{\partial \mathrm{z}}\left[\cos ^{-1}\left(\frac{z}{r}\right)\right]
$$

Again, we use the definition for the derivative of the arc cosine.

$$
\frac{\mathrm{d}}{\mathrm{dz}}\left(\cos ^{-1}(f)\right)=-\frac{\frac{\mathrm{d}(f)}{\mathrm{dz}}}{\sqrt{1-\mathrm{f}^{2}}}
$$

Applying it to our case, we have

$$
\frac{\partial}{\partial z}\left(\cos ^{-1}\left(\frac{z}{r}\right)\right)=-\frac{\frac{\partial}{\partial z}\left(\frac{z}{r}\right)}{\sqrt{1-\left(\frac{z}{r}\right)^{2}}}
$$


We know that $\frac{\mathrm{z}}{\mathrm{r}}=\cos \theta$; then the denominator is $\sqrt{1-\cos ^{2} \theta}$. We know that $\sin \theta=\sqrt{1-\cos ^{2} \theta}$; so, from now on, we can write

$$
\frac{\partial}{\partial z}\left(\cos ^{-1}\left(\frac{z}{r}\right)\right)=-\frac{\frac{\partial}{\partial z}\left(\frac{z}{r}\right)}{\sin \theta}
$$

In the previous cases, $\mathrm{z}$ was a constant and left the derivative. Now, we have that $\mathrm{z}$ is a variable, so we have to use the quotient rule for derivatives. Let us remember the quotient rule.

Let $\mathrm{f}(\mathrm{x})=\frac{\mathrm{g}(\mathrm{x})}{\mathrm{h}(\mathrm{x})}$ then its derivative is: $\frac{\mathrm{d}}{\mathrm{dx}}(\mathrm{f}(\mathrm{x}))=\frac{\mathrm{g}^{\prime}(\mathrm{x}) \mathrm{h}(\mathrm{x})-\mathrm{g}(\mathrm{x}) \mathrm{h}^{\prime}(\mathrm{x})}{\mathrm{h}(\mathrm{x})^{2}}$.

So, for our case, we have

$$
\frac{\partial}{\partial z}\left(\frac{z}{r}\right)=\frac{\left(z^{\prime}\right)(r)-(z)\left(r^{\prime}\right)}{r^{2}}=\frac{r-(z)\left[\frac{\partial}{\partial z}\left(\sqrt{\mathrm{x}^{2}+\mathrm{y}^{2}+\mathrm{z}^{2}}\right)\right]}{\mathrm{x}^{2}+\mathrm{y}^{2}+\mathrm{z}^{2}}
$$

Let us perform separately the only partial derivative that appears in the numerator:

$$
\frac{\partial}{\partial z}\left(\sqrt{x^{2}+y^{2}+z^{2}}\right)
$$

We use the following variable change: $u=x^{2}+y^{2}+z^{2}$, then:

$$
\begin{aligned}
& \frac{\partial}{\partial \mathrm{z}}=\frac{\partial(\sqrt{\mathrm{u}})}{\mathrm{du}} \frac{\partial \mathrm{u}}{\partial \mathrm{z}} \\
& \frac{\partial(\sqrt{\mathrm{u}})}{\partial \mathrm{u} \mathrm{u} u}=\frac{1}{2} \mathrm{u}^{-\frac{1}{2}} \\
& \frac{\partial \mathrm{u}}{\partial \mathrm{z}}=2 \mathrm{z} \\
& \frac{\partial(\sqrt{\mathrm{u}})}{\mathrm{du}} \frac{\partial \mathrm{u}}{\partial \mathrm{z}}=\frac{1}{2} \mathrm{u}^{-\frac{1}{2}}(2 \mathrm{z}) \\
& \frac{\partial}{\partial z}\left(\sqrt{x^{2}+y^{2}+z^{2}}\right)=\frac{z}{\sqrt{x^{2}+y^{2}+z^{2}}}=\frac{z}{r}
\end{aligned}
$$

We insert this last result in the quotient expression to have:

$$
\frac{\partial}{\partial z}\left(\frac{z}{r}\right)=\frac{r-(z)\left(\frac{z}{r}\right)}{r^{2}}=\frac{r-\frac{z^{2}}{r}}{r^{2}}
$$

Remember that $\mathrm{z}=\mathrm{r} \cos \theta$; thus,

$$
\frac{\partial}{\partial z}\left(\frac{z}{r}\right)=\frac{r-\frac{z^{2}}{r}}{r^{2}}=\frac{r-\frac{r^{2} \cos ^{2} \theta}{r}}{r^{2}}=\frac{r-r \cos ^{2} \theta}{r^{2}}
$$

Dividing the numerator and denominator by $\mathrm{r}$, we have

$$
\frac{\partial}{\partial z}\left(\frac{z}{r}\right)=\frac{1-\cos ^{2} \theta}{r}=\frac{\sin ^{2} \theta}{r}
$$

We insert this last result in the expression for the original derivative of the arcsine and we have:

$$
\frac{\partial}{\partial z}\left(\cos ^{-1}\left(\frac{z}{r}\right)\right)=-\frac{\frac{\partial}{\partial z}\left(\frac{z}{r}\right)}{\sin \theta}=-\frac{\frac{\sin ^{2} \theta}{r}}{\sin \theta}=-\frac{\sin \theta}{r}
$$

Which it turns out that:

$$
\frac{\partial \theta}{\partial z}=-\frac{\sin \theta}{r}
$$

Now we should perform a similar procedure for variable $\phi$ derivatives.

Let's start with $\frac{\partial \phi}{\partial x}$

$$
\frac{\partial \phi}{\partial x}=\frac{\partial}{\partial x}\left[\tan ^{-1}\left(\frac{y}{x}\right)\right]
$$


We make a change of variable: $u=\frac{y}{x}$, then:

$$
\frac{\partial \phi}{\partial \mathrm{x}}=\frac{\partial}{\partial \mathrm{x}}\left[\tan ^{-1}\left(\frac{\mathrm{y}}{\mathrm{x}}\right)\right]=\frac{\partial}{\partial \mathrm{u}}\left[\tan ^{-1}(\mathrm{u})\right] \frac{\partial \mathrm{u}}{\partial \mathrm{x}}
$$

For $\frac{\partial}{\partial u}\left[\tan ^{-1}(u)\right]$, let us remember that there is a formula for the derivative of the tangent arc of a function, which is

$$
\frac{\mathrm{d}}{\mathrm{dx}}\left[\tan ^{-1}(\mathrm{x})\right]=\frac{1}{\mathrm{x}^{2}+1}
$$

So, in our case, it is applied directly.

$$
\frac{\partial}{\partial \mathrm{u}}\left[\tan ^{-1}(\mathrm{u})\right]=\frac{1}{\mathrm{u}^{2}+1}
$$

Meanwhile $\frac{\partial u}{\partial x}=\frac{\partial}{\partial x}\left[\frac{y}{x}\right]=y \frac{\partial}{\partial x}\left(x^{-1}\right)=-y\left(x^{-2}\right)=-\frac{y}{x^{2}}$

Substituting both results in our original expression we have:

$$
\begin{gathered}
\frac{\partial \phi}{\partial x}=\frac{\partial}{\partial x}\left[\tan ^{-1}\left(\frac{y}{x}\right)\right]=\frac{\partial}{\partial u}\left[\tan ^{-1}(u)\right] \frac{\partial u}{\partial x}=\left[\frac{1}{\left(\frac{y}{x}\right)^{2}+1}\right]\left[\frac{-y}{x^{2}}\right] \\
\frac{\partial \phi}{\partial x}=-\frac{y}{x^{2}\left(1+\frac{y^{2}}{x^{2}}\right)}=-\frac{y}{x^{2}+y^{2}}
\end{gathered}
$$

Recall again that $y=r \sin \theta \sin \phi$ and $x=r \sin \theta \cos \phi$, then we have:

$$
\frac{\partial \phi}{\partial x}=-\frac{y}{x^{2}+y^{2}}=-\frac{r \sin \theta \sin \phi}{\left(r^{2} \sin \theta^{2} \theta \cos ^{2} \phi\right)+\left(r^{2} \sin ^{2} \theta \sin ^{2} \phi\right)}
$$

Factoring the denominator,

$$
\frac{\partial \phi}{\partial x}=-\frac{r \sin \theta \sin \phi}{r^{2} \sin ^{2} \theta\left(\cos ^{2} \phi+\sin ^{2} \phi\right)}
$$

where $\cos ^{2} \phi+\sin ^{2} \phi=1$

$$
\frac{\partial \phi}{\partial x}=-\frac{r \sin \theta \sin \phi}{r^{2} \sin ^{2} \theta}=-\frac{\sin \phi}{r \sin \theta}
$$

So, we can write the following:

$$
\frac{\partial \phi}{\partial x}=-\frac{\sin \phi}{r \sin \theta}
$$

We continue now with $\frac{\partial \phi}{\partial y}$.

$$
\frac{\partial \phi}{\partial y}=\frac{\partial}{\partial y}\left[\tan ^{-1}\left(\frac{y}{x}\right)\right]
$$

Again, we make a change of variable $u=\frac{y}{x}$, to have:

$$
\begin{gathered}
\frac{\partial \phi}{\partial y}=\frac{\partial}{\partial y}\left[\tan ^{-1}\left(\frac{y}{x}\right)\right]=\frac{\partial}{\partial u}\left[\tan ^{-1}(u)\right] \frac{\partial u}{\partial y} \\
\frac{\partial}{\partial u}\left[\tan ^{-1}(u)\right]=\frac{1}{u^{2}+1} \\
\frac{\partial u}{\partial y}=\frac{1}{x} \frac{\partial(y)}{\partial y}=\frac{1}{x}
\end{gathered}
$$


Substituting both results in our original expression:

$$
\begin{gathered}
\frac{\partial \phi}{\partial y}=\frac{\partial}{\partial y}\left[\tan ^{-1}\left(\frac{y}{x}\right)\right]=\frac{\partial}{\partial u}\left[\tan ^{-1}(u)\right] \frac{\partial u}{\partial y}=\left[\frac{1}{\left(\frac{y}{x}\right)^{2}+1}\right]\left[\frac{1}{x}\right] \\
\frac{\partial \phi}{\partial y}=-\frac{1}{x\left(1+\frac{y^{2}}{x^{2}}\right)}=\frac{1}{x+\frac{y^{2}}{x}}
\end{gathered}
$$

Multiplying by $\mathrm{x}$ numerator and denominator,

$$
\frac{\partial \phi}{\partial y}=\frac{x}{x^{2}+y^{2}}
$$

Recall again that $y=r \sin \theta \sin \phi$ and $x=r \sin \theta \cos \phi$,

$$
\frac{\partial \phi}{\partial y}=-\frac{x}{x^{2}+y^{2}}=\frac{r \sin \theta \cos \phi}{\left(r^{2} \sin ^{2} \theta \cos ^{2} \phi\right)+\left(r^{2} \sin ^{2} \theta \sin ^{2} \phi\right)}
$$

Factoring the denominator,

$$
\frac{\partial \phi}{\partial y}=\frac{r \sin \theta \cos \phi}{r^{2} \sin ^{2} \theta\left(\cos ^{2} \phi+\sin ^{2} \phi\right)}
$$

where $\cos ^{2} \phi+\sin ^{2} \phi=1$.

$$
\frac{\partial \phi}{\partial y}=\frac{r \sin \theta \cos \phi}{r^{2} \sin ^{2} \theta}=\frac{\cos \phi}{r \sin \theta}
$$

Therefore, we have

$$
\frac{\partial \phi}{\partial y}=\frac{\cos \phi}{\mathrm{r} \sin \theta}
$$

Finally, for $\frac{\partial \phi}{\partial z}$, we have

$$
\frac{\partial \phi}{\partial z}=\frac{\partial}{\partial z}\left[\tan ^{-1}\left(\frac{y}{x}\right)\right]
$$

Since the variable $z$ does not appear in the expression, then $\tan ^{-1}\left(\frac{y}{x}\right)$ is constant; therefore, we can write

$$
\frac{\partial \phi}{\partial z}=0
$$

So far, we calculated the nine derivatives of the "new" variables with respect to the "old" ones. We present them together in Equation (3).

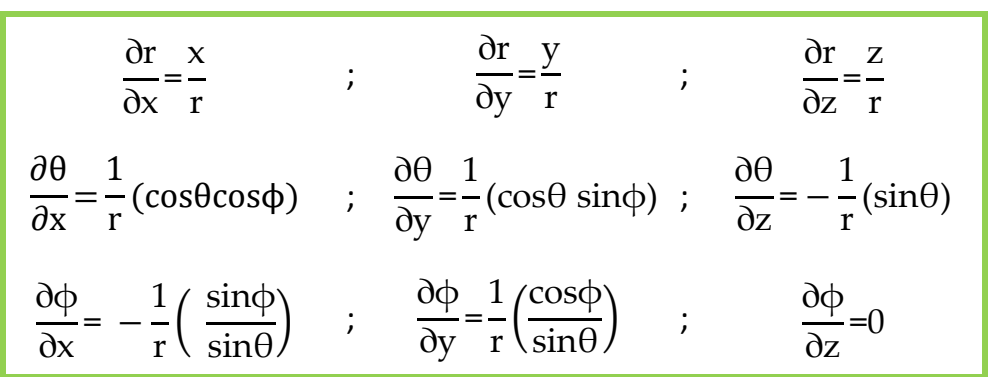

We should now use the nine equations presented in Equation (3) into the Equation (1). Let us start with $\frac{\partial f}{\partial x}$.

$$
\frac{\partial \mathrm{f}}{\partial \mathrm{x}}=\frac{\partial \mathrm{f}}{\partial \mathrm{r}} \frac{\partial \mathrm{r}}{\partial \mathrm{x}}+\frac{\partial \mathrm{f}}{\partial \theta} \frac{\partial \theta}{\partial \mathrm{x}}+\frac{\partial \mathrm{f}}{\partial \phi} \frac{\partial \phi}{\partial \mathrm{x}}
$$


Substituting $\frac{\partial \mathrm{r}}{\partial \mathrm{x}} ; \frac{\partial \theta}{\partial \mathrm{x}} ; \frac{\partial \phi}{\partial \mathrm{x}}$, we have:

$$
\frac{\partial \mathrm{f}}{\partial \mathrm{x}}=\frac{\mathrm{x}}{\mathrm{r}} \frac{\partial \mathrm{f}}{\partial \mathrm{r}}+\frac{1}{\mathrm{r}}(\cos \theta \cos \phi) \frac{\partial \mathrm{f}}{\partial \theta}-\frac{1}{\mathrm{r}}\left(\frac{\sin \phi}{\sin \theta}\right) \frac{\partial \mathrm{f}}{\partial \phi}
$$

Let us notice that, on the right side of the above equation, we still have one of the "old" variables, which is $x$. Therefore, we need to replace it using the coordinate transformation that we presented in Equation (2).

$$
\frac{\partial \mathrm{f}}{\partial \mathrm{x}}=\sin \theta \cos \phi \frac{\partial \mathrm{f}}{\partial \mathrm{r}}+\frac{1}{\mathrm{r}}(\cos \theta \cos \phi) \frac{\partial \mathrm{f}}{\partial \theta}-\frac{1}{\mathrm{r}}\left(\frac{\sin \phi}{\sin \theta}\right) \frac{\partial \mathrm{f}}{\partial \phi}
$$

In the last expression for $\frac{\partial f}{\partial x}$, we derive $\mathrm{f}$ with respect to $\mathrm{x}$. Now, we can express it as an operator, so that it "operates" on whatever function appearing to its right. For this, it is only necessary to remove the function $\mathrm{f}$.

$$
\frac{\partial}{\partial \mathrm{x}}=\sin \theta \cos \phi \frac{\partial}{\partial \mathrm{r}}+\frac{1}{\mathrm{r}} \cos \theta \cos \phi \frac{\partial}{\partial \theta}-\frac{1}{\mathrm{r}} \frac{\sin \phi}{\sin \theta} \frac{\partial}{\partial \phi}
$$

Let us continue with $\frac{\partial f}{\partial y}$.

$$
\frac{\partial \mathrm{f}}{\partial \mathrm{y}}=\frac{\partial \mathrm{f}}{\partial \mathrm{r}} \frac{\partial \mathrm{r}}{\partial \mathrm{y}}+\frac{\partial \mathrm{f}}{\partial \theta} \frac{\partial \theta}{\partial \mathrm{y}}+\frac{\partial \mathrm{f}}{\partial \phi} \frac{\partial \phi}{\partial \mathrm{y}}
$$

Substituting $\frac{\partial \mathrm{r}}{\partial \mathrm{y}} ; \frac{\partial \theta}{\partial \mathrm{y}} ; \frac{\partial \phi}{\partial \mathrm{y}}$, we have

$$
\frac{\partial \mathrm{f}}{\partial \mathrm{y}}=\frac{\mathrm{y}}{\mathrm{r}} \frac{\partial \mathrm{f}}{\partial \mathrm{r}}+\frac{1}{\mathrm{r}}(\cos \theta \sin \phi) \frac{\partial \mathrm{f}}{\partial \theta}+\frac{1}{\mathrm{r}}\left(\frac{\cos \phi}{\sin \theta}\right) \frac{\partial \mathrm{f}}{\partial \phi}
$$

Let us replace $y=r \sin \theta \sin \phi$.

$$
\frac{\partial \mathrm{f}}{\partial \mathrm{y}}=\frac{\mathrm{r} \sin \theta \sin \phi}{\mathrm{r}} \frac{\partial \mathrm{f}}{\partial \mathrm{r}}+\frac{1}{\mathrm{r}}(\cos \theta \sin \phi) \frac{\partial \mathrm{f}}{\partial \theta}+\frac{1}{\mathrm{r}}\left(\frac{\cos \phi}{\sin \theta}\right) \frac{\partial \mathrm{f}}{\partial \phi}
$$

Converting this last expression to an operator,

$$
\frac{\partial}{\partial y}=\sin \theta \sin \phi \frac{\partial}{\partial r}+\frac{1}{r} \cos \theta \sin \phi \frac{\partial}{\partial \theta}+\frac{1}{r} \frac{\cos \phi}{\sin \theta} \frac{\partial}{\partial \phi}
$$

We repeat the procedure for $\frac{\partial f}{\partial z}$.

$$
\frac{\partial \mathrm{f}}{\partial \mathrm{z}}=\frac{\partial \mathrm{f}}{\partial \mathrm{r}} \frac{\partial \mathrm{r}}{\partial \mathrm{z}}+\frac{\partial \mathrm{f}}{\partial \theta} \frac{\partial \theta}{\partial \mathrm{z}}+\frac{\partial \mathrm{f}}{\partial \phi} \frac{\partial \phi}{\partial \mathrm{z}}
$$

Substituting $\frac{\partial \mathrm{r}}{\partial \mathrm{z}} ; \frac{\partial \theta}{\partial \mathrm{z}} ; \frac{\partial \phi}{\partial \mathrm{z}}$, we have

$$
\frac{\partial \mathrm{f}}{\partial \mathrm{z}}=\frac{\mathrm{z}}{\mathrm{r}} \frac{\partial \mathrm{f}}{\partial \mathrm{r}}-\frac{1}{\mathrm{r}} \sin \theta \frac{\partial \mathrm{f}}{\partial \theta}+0
$$

Replacing $\mathrm{z}$ with $\mathrm{z}=\mathrm{r} \cos \theta$, we have

$$
\frac{\partial \mathrm{f}}{\partial \mathrm{z}}=\cos \theta \frac{\partial \mathrm{f}}{\partial \mathrm{r}}-\frac{1}{\mathrm{r}} \sin \theta \frac{\partial \mathrm{f}}{\partial \theta}
$$

Converting expression to operator,

$$
\frac{\partial}{\partial z}=\cos \theta \frac{\partial}{\partial r}-\frac{1}{r} \sin \theta \frac{\partial}{\partial \theta}
$$


Now, writing the three operators $\frac{\partial}{\partial x} ; \frac{\partial}{\partial y} ; \frac{\partial}{\partial z}$,

$$
\begin{gathered}
\frac{\partial}{\partial x}=\sin \theta \cos \phi \frac{\partial}{\partial r}+\frac{1}{r} \cos \theta \cos \phi \frac{\partial}{\partial \theta}-\frac{1}{r} \frac{\sin \phi}{\sin \theta} \frac{\partial}{\partial \phi} \\
\frac{\partial}{\partial y}=\sin \theta \sin \phi \frac{\partial}{\partial r}+\frac{1}{r} \cos \theta \sin \phi \frac{\partial}{\partial \theta}+\frac{1}{r} \frac{\cos \phi}{\sin \theta} \frac{\partial}{\partial \phi} \\
\frac{\partial}{\partial z}=\cos \theta \frac{\partial}{\partial r}-\frac{1}{r} \sin \theta \frac{\partial}{\partial \theta}
\end{gathered}
$$

Let us recall the Laplacian operator in rectangular coordinates:

$$
\nabla^{2}=\frac{\partial^{2}}{\partial x^{2}}+\frac{\partial^{2}}{\partial y^{2}}+\frac{\partial^{2}}{\partial z^{2}}
$$

The Laplacian operates "twice" on the scalar function. We can interpret it as a "multiplication" of two Nabla operators.

We use the same concept to obtain the Laplacian operator in spherical coordinates. To make this procedure easily digestible, we divided it in sections.

Section corresponding to $\frac{\partial}{\partial x}\left(\frac{\partial}{\partial x}\right)$.

$$
\begin{gathered}
\frac{\partial}{\partial x}\left(\frac{\partial}{\partial x}\right)=\left[\sin \theta \cos \phi \frac{\partial}{\partial r}+\frac{1}{r} \cos \theta \cos \phi \frac{\partial}{\partial \theta}-\frac{1}{r} \frac{\sin \phi}{\sin \theta} \frac{\partial}{\partial \phi}\right] \\
{\left[\sin \theta \cos \phi \frac{\partial}{\partial r}+\frac{1}{r} \cos \theta \cos \phi \frac{\partial}{\partial \theta}-\frac{1}{r} \frac{\sin \phi}{\sin \theta} \frac{\partial}{\partial \phi}\right]}
\end{gathered}
$$

We must operate term by term. Observe that since we have three terms, in each $\frac{\partial}{\partial x}$ we will have nine terms at the end. Let us proceed.

First-term operating on the first term $\left(\right.$ for $\left.\frac{\partial}{\partial x}\left(\frac{\partial}{\partial x}\right)\right)$.

$$
\left(\sin \theta \cos \phi \frac{\partial}{\partial \mathrm{r}}\right)\left(\sin \theta \cos \phi \frac{\partial}{\partial \mathrm{r}}\right)
$$

$\sin \theta \cos \phi$ are constant when "operated" by $\frac{\partial}{\partial r}$. Therefore, the result is:

$$
\sin ^{2} \theta \cos ^{2} \phi \frac{\partial^{2}}{\partial \mathrm{r}^{2}}
$$

First-term operating on the second term $\left(\right.$ for $\left.\frac{\partial}{\partial x}\left(\frac{\partial}{\partial x}\right)\right)$.

$$
\left(\sin \theta \cos \phi \frac{\partial}{\partial \mathrm{r}}\right)\left(\frac{1}{\mathrm{r}} \cos \theta \cos \phi \frac{\partial}{\partial \theta}\right)
$$

Let us see that a $\frac{1}{r}$ appears in the second term; therefore, we have to treat this second term as a multiplication of functions, as follows:

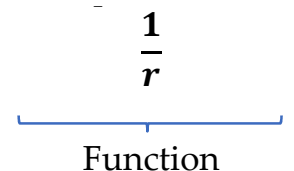

(1)

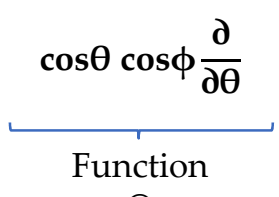

(2) 
Accordingly, we will use the product rule in order to operate the first term on the second.

$$
\begin{gathered}
(\mathrm{f} \cdot \mathrm{g})^{\prime}=\mathrm{f}^{\prime} \cdot \mathrm{g}+\mathrm{f} \cdot \mathrm{g}^{\prime} \\
\sin \theta \cos \phi \frac{\partial}{\partial \mathrm{r}}\left[\frac{1}{\mathrm{r}} \cos \theta \cos \phi \frac{\partial}{\partial \theta}\right]
\end{gathered}
$$

Let us perform just the derivative instruction $\frac{\partial}{\partial \mathrm{r}}\left[\frac{1}{\mathrm{r}} \cos \theta \cos \phi \frac{\partial}{\partial \theta}\right]$ :

$$
\frac{\partial}{\partial \mathrm{r}}\left[\frac{1}{\mathrm{r}} \cos \theta \cos \phi \frac{\partial}{\partial \theta}\right]=\left[\left(-\frac{1}{\mathrm{r}^{2}}\right) \cos \theta \cos \phi \frac{\partial}{\partial \theta}+\frac{1}{\mathrm{r}} \cos \theta \cos \phi \frac{\partial^{2}}{\partial \mathrm{r} \partial \theta}\right]
$$

We insert the result of our derivative in the original expression to have:

$$
\sin \theta \cos \phi\left[\left(-\frac{1}{\mathrm{r}^{2}}\right) \cos \theta \cos \phi \frac{\partial}{\partial \theta}+\frac{1}{\mathrm{r}} \cos \theta \cos \phi \frac{\partial^{2}}{\partial \mathrm{r} \partial \theta}\right]
$$

Expanding, we have:

$$
-\frac{1}{\mathrm{r}^{2}} \cos \theta \cos ^{2} \phi \sin \theta \frac{\partial}{\partial \theta}+\frac{1}{\mathrm{r}} \cos \theta \cos ^{2} \phi \sin \theta \frac{\partial^{2}}{\partial \mathrm{r} \partial \theta}
$$

And finally factoring, we have that the result of the first term operating on the second term for $\frac{\partial}{\partial x}\left(\frac{\partial}{\partial x}\right)$ is:

$$
-\frac{1}{\mathrm{r}^{2}} \cos \theta \cos ^{2} \phi \sin \theta\left(\frac{\partial}{\partial \theta}-\mathrm{r} \frac{\partial^{2}}{\partial \mathrm{r} \partial \theta}\right)
$$

First-term operating on the third term $\left(\right.$ for $\left.\frac{\partial}{\partial x}\left(\frac{\partial}{\partial x}\right)\right)$.

$$
\left(\sin \theta \cos \phi \frac{\partial}{\partial \mathrm{r}}\right)\left(-\frac{1}{\mathrm{r}} \frac{\sin \phi}{\sin \theta} \frac{\partial}{\partial \phi}\right)
$$

Again, we have to treat the third term as a multiplication of functions, as follows:

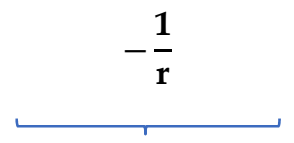

Function

(1)

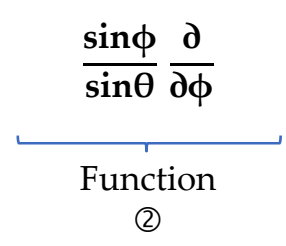

$\sin \theta \cos \phi \frac{\partial}{\partial \mathrm{r}}\left[-\frac{1}{\mathrm{r}} \frac{\sin \phi}{\sin \theta} \frac{\partial}{\partial \phi}\right]$

Performing just the derivative instruction:

$$
\frac{\partial}{\partial \mathrm{r}}\left[-\frac{1}{\mathrm{r}} \frac{\sin \phi}{\sin \theta} \frac{\partial}{\partial \phi}\right]=\left[\left(\frac{1}{\mathrm{r}^{2}}\right) \frac{\sin \phi}{\sin \theta} \frac{\partial}{\partial \phi}-\frac{1}{\mathrm{r}} \frac{\sin \phi}{\sin \theta} \frac{\partial^{2}}{\partial \mathrm{r} \partial \phi}\right]
$$

Inserting the result of our derivative in the original expression

$$
\sin \theta \cos \phi\left[\left(\frac{1}{\mathrm{r}^{2}}\right) \frac{\sin \phi}{\sin \theta} \frac{\partial}{\partial \phi}-\frac{1}{\mathrm{r}} \frac{\sin \phi}{\sin \theta} \frac{\partial^{2}}{\partial \mathrm{r} \partial \phi}\right]
$$

Expanding:

$$
\frac{1}{\mathrm{r}^{2}} \cos \phi \sin \phi \frac{\partial}{\partial \phi}-\frac{1}{\mathrm{r}} \cos \phi \sin \phi \frac{\partial^{2}}{\partial \mathrm{r} \partial \phi}
$$


Factoring,

$$
\frac{1}{\mathrm{r}^{2}} \cos \phi \sin \phi\left(\frac{\partial}{\partial \phi}-\mathrm{r} \frac{\partial^{2}}{\partial \mathrm{r} \partial \phi}\right)
$$

Second-term operating on the first term $\left(\right.$ for $\left.\frac{\partial}{\partial x}\left(\frac{\partial}{\partial x}\right)\right)$.

$$
\left(\frac{1}{\mathrm{r}} \cos \theta \cos \phi \frac{\partial}{\partial \phi}\right)\left(\sin \theta \cos \phi \frac{\partial}{\partial \mathrm{r}}\right)
$$

Please remember that at this moment, we are "operating" the second term on the first term. In other words, in this particular case, the first term belonging to $\frac{\partial}{\partial x}$ appears to the right of the second term belonging to $\frac{\partial}{\partial x}$. Let us treat the first term as a multiplication of functions, as follows:

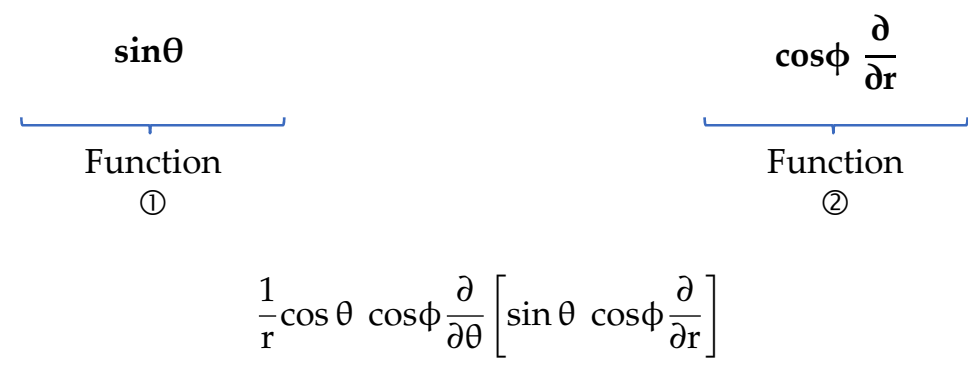

Performing just the derivative instruction:

$$
\frac{\partial}{\partial \theta}\left[\sin \theta \cos \phi \frac{\partial}{\partial r}\right]=\left[\cos \theta \cos \phi \frac{\partial}{\partial r}+\sin \theta \cos \phi \frac{\partial^{2}}{\partial \theta \partial r}\right]
$$

Inserting the result of our derivative in the original expression

$$
\frac{1}{r} \cos \theta \cos \phi\left[\cos \theta \cos \phi \frac{\partial}{\partial r}+\sin \theta \cos \phi \frac{\partial^{2}}{\partial \theta \partial r}\right]
$$

Expanding,

$$
\frac{1}{r} \cos ^{2} \theta \cos ^{2} \phi \frac{\partial}{\partial r}+\frac{1}{r} \cos \theta \cos ^{2} \phi \sin \theta \frac{\partial^{2}}{\partial \theta \partial r}
$$

Factoring,

$$
\frac{1}{\mathrm{r}} \cos \theta \cos ^{2} \phi\left(\cos \theta \frac{\partial}{\partial \mathrm{r}}+\sin \theta \frac{\partial^{2}}{\partial \theta \partial \mathrm{r}}\right)
$$

Second-term operating on the second term $\left(\right.$ for $\left.\frac{\partial}{\partial x}\left(\frac{\partial}{\partial x}\right)\right)$.

$$
\left(\frac{1}{\mathrm{r}} \cos \theta \cos \phi \frac{\partial}{\partial \theta}\right)\left(\frac{1}{\mathrm{r}} \cos \theta \cos \phi \frac{\partial}{\partial \theta}\right)
$$

In the second term, we have a multiplication of functions

$$
\frac{1}{\mathrm{r}} \cos \theta \quad \cos \phi \frac{\partial}{\partial \theta}
$$

Function

Function

(1)

$$
\frac{1}{\mathrm{r}} \cos \theta \cos \phi \frac{\partial}{\partial \theta}\left[\frac{1}{\mathrm{r}} \cos \theta \cos \phi \frac{\partial}{\partial \theta}\right]
$$


Performing just the derivative instruction:

$$
\frac{\partial}{\partial \theta}\left[\frac{1}{\mathrm{r}} \cos \theta \cos \phi \frac{\partial}{\partial \theta}\right]=\left[-\frac{1}{\mathrm{r}} \sin \theta \cos \phi \frac{\partial}{\partial \theta}+\frac{1}{\mathrm{r}} \cos \theta \cos \phi \frac{\partial^{2}}{\partial \theta^{2}}\right]
$$

Inserting the result of our derivative in the original expression

$$
\frac{1}{\mathrm{r}} \cos \theta \cos \phi\left[-\frac{1}{\mathrm{r}} \sin \theta \cos \phi \frac{\partial}{\partial \theta}+\frac{1}{\mathrm{r}} \cos \theta \cos \phi \frac{\partial^{2}}{\partial \theta^{2}}\right]
$$

Expanding,

$$
-\frac{1}{\mathrm{r}^{2}} \cos \theta \cos ^{2} \phi \sin \theta \frac{\partial}{\partial \theta}+\frac{1}{\mathrm{r}^{2}} \cos ^{2} \theta \cos ^{2} \phi \frac{\partial^{2}}{\partial \theta^{2}}
$$

Factoring,

$$
\frac{1}{\mathrm{r}^{2}} \cos \theta \cos ^{2} \phi\left(-\sin \theta \frac{\partial}{\partial \theta}+\cos \theta \frac{\partial^{2}}{\partial \theta^{2}}\right)
$$

Second-term operating on the third term $\left(\right.$ for $\left.\frac{\partial}{\partial x}\left(\frac{\partial}{\partial x}\right)\right)$.

$$
\left(\frac{1}{\mathrm{r}} \cos \theta \cos \phi \frac{\partial}{\partial \theta}\right)\left(-\frac{1}{\mathrm{r}} \frac{\sin \phi}{\sin \theta} \frac{\partial}{\partial \phi}\right)
$$

The third term is a multiplication of functions:

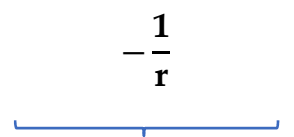

Function

(1) $\frac{\sin \phi}{\sin \theta} \frac{\partial}{\partial \phi}$

Function

(2)

$$
\frac{1}{\mathrm{r}} \cos \theta \cos \phi \frac{\partial}{\partial \theta}\left[-\frac{1}{\mathrm{r}} \frac{\sin \phi}{\sin \theta} \frac{\partial}{\partial \phi}\right]
$$

Performing just the derivative instruction:

$$
\frac{\partial}{\partial \theta}\left[-\frac{1}{\mathrm{r}} \frac{\sin \phi}{\sin \theta} \frac{\partial}{\partial \phi}\right]
$$

Again, we have to use the product rule. However, for this, we have first to derive $\frac{d}{d \theta}\left(\frac{1}{\sin \theta}\right)$.

Making a change of variable $u=\sin \theta$, we have

$$
\begin{gathered}
\frac{d}{d u}\left(\frac{1}{u}\right) \frac{d u}{d \theta} \\
\frac{d}{d u}\left(\frac{1}{u}\right)=-\frac{1}{u^{2}} \quad \frac{d u}{d \theta}=\frac{d(\sin \theta)}{d \theta}=\cos \theta \\
\frac{d}{d \theta}\left(\frac{1}{\sin \theta}\right)=\frac{d}{d u}\left(\frac{1}{u}\right) \frac{d u}{d \theta}=-\frac{1}{u^{2}}(\cos \theta)=-\frac{1}{\sin ^{2} \theta} \cos \theta \\
\frac{d}{d \theta}\left(\frac{1}{\sin \theta}\right)=-\frac{\cos \theta}{\sin ^{2} \theta}
\end{gathered}
$$


Going back to the derivative instruction, we have

$$
\begin{gathered}
\frac{\partial}{\partial \theta}\left[-\frac{1}{\mathrm{r}} \frac{\sin \phi}{\sin \theta} \frac{\partial}{\partial \phi}\right]=\left[-\frac{1}{\mathrm{r}} \sin \phi\left(-\frac{\cos \theta}{\sin ^{2} \theta}\right) \frac{\partial}{\partial \phi}-\frac{1}{\mathrm{r}} \frac{\sin \phi}{\sin \theta} \frac{\partial^{2}}{\partial \theta \partial \phi}\right] \\
\frac{\partial}{\partial \theta}\left[-\frac{1}{\mathrm{r}} \frac{\sin \phi}{\sin \theta} \frac{\partial}{\partial \phi}\right]=\left[\frac{1}{\mathrm{r}} \frac{\sin \phi \cos \theta}{\sin ^{2} \theta} \frac{\partial}{\partial \phi}-\frac{1}{\mathrm{r}} \frac{\sin \phi}{\sin \theta} \frac{\partial^{2}}{\partial \theta \partial \phi}\right]
\end{gathered}
$$

Inserting the result of our derivative in the original expression

$$
\frac{1}{\mathrm{r}} \cos \theta \cos \phi\left[\frac{1}{\mathrm{r}} \frac{\sin \phi \cos \theta}{\sin ^{2} \theta} \frac{\partial}{\partial \phi}-\frac{1}{\mathrm{r}} \frac{\sin \phi}{\sin \theta} \frac{\partial^{2}}{\partial \theta \partial \phi}\right]
$$

Expanding,

$$
\frac{1}{\mathrm{r}^{2}} \frac{\cos \phi \sin \phi \cos ^{2} \theta}{\sin ^{2} \theta} \frac{\partial}{\partial \phi}-\frac{1}{\mathrm{r}^{2}} \frac{\cos \phi \sin \phi \cos \theta}{\sin \theta} \frac{\partial^{2}}{\partial \theta \partial \phi}
$$

Factoring,

$$
\frac{1}{\mathrm{r}^{2}} \frac{\cos \phi \sin \phi \cos \theta}{\sin \theta}\left(\frac{\cos \theta}{\sin \theta} \frac{\partial}{\partial \phi}-\frac{\partial^{2}}{\partial \theta \partial \phi}\right)
$$

Third-term operating on the first term $\left(\right.$ for $\left.\frac{\partial}{\partial x}\left(\frac{\partial}{\partial x}\right)\right)$.

$$
\left(-\frac{1}{\mathrm{r}} \frac{\sin \phi}{\sin \theta} \frac{\partial}{\partial \phi}\right)\left(\sin \theta \cos \phi \frac{\partial}{\partial \mathrm{r}}\right)
$$

The first term is a multiplication of two functions:

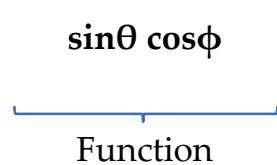

(1)

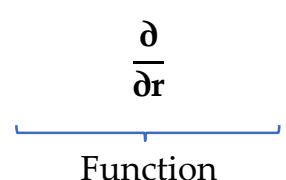

(2)

$$
-\frac{1}{\mathrm{r}} \frac{\sin \phi}{\sin \theta} \frac{\partial}{\partial \phi}\left[\sin \theta \cos \phi \frac{\partial}{\partial \mathrm{r}}\right]
$$

Performing just the derivative instruction:

$$
\frac{\partial}{\partial \phi}\left[\sin \theta \cos \phi \frac{\partial}{\partial r}\right]=\left[-\sin \theta \sin \phi \frac{\partial}{\partial r}+\sin \theta \cos \phi \frac{\partial^{2}}{\partial \phi \partial r}\right]
$$

Inserting the result of our derivative in the original expression

$$
-\frac{1}{r} \frac{\sin \phi}{\sin \theta}\left[-\sin \theta \sin \phi \frac{\partial}{\partial r}+\sin \theta \cos \phi \frac{\partial^{2}}{\partial \phi \partial r}\right]
$$

Expanding,

$$
\frac{1}{r} \sin ^{2} \phi \frac{\partial}{\partial r}-\frac{1}{r} \cos \phi \sin \phi \frac{\partial^{2}}{\partial \phi \partial r}
$$

Factoring,

$$
-\frac{1}{r} \sin \phi\left(-\sin \phi \frac{\partial}{\partial r}+\cos \phi \frac{\partial^{2}}{\partial \phi \partial r}\right)
$$


Third-term operating on the second term $\left(\right.$ for $\left.\frac{\partial}{\partial x}\left(\frac{\partial}{\partial x}\right)\right)$.

$$
\left(-\frac{1}{\mathrm{r}} \frac{\sin \phi}{\sin \theta} \frac{\partial}{\partial \phi}\right)\left(\frac{1}{\mathrm{r}} \cos \theta \cos \phi \frac{\partial}{\partial \theta}\right)
$$

The second term is a multiplication of two functions:

$$
\begin{aligned}
& \frac{1}{r} \cos \theta \cos \phi \\
& \text { Function } \\
& \text { (1) } \\
& -\frac{1}{r} \frac{\sin \phi}{\sin \theta} \frac{\partial}{\partial \phi}\left[\frac{1}{r} \cos \theta \cos \phi \frac{\partial}{\partial \theta}\right]
\end{aligned}
$$

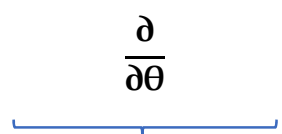

Performing just the derivative instruction:

$$
\frac{\partial}{\partial \phi}\left[\frac{1}{\mathrm{r}} \cos \theta \cos \phi \frac{\partial}{\partial \theta}\right]=\left[-\frac{1}{\mathrm{r}} \cos \theta \sin \phi \frac{\partial}{\partial \theta}+\frac{1}{\mathrm{r}} \cos \theta \cos \phi \frac{\partial^{2}}{\partial \phi \partial \theta}\right]
$$

Inserting the result of our derivative in the original expression

$$
-\frac{1}{\mathrm{r}} \frac{\sin \phi}{\sin \theta}\left[-\frac{1}{\mathrm{r}} \cos \theta \sin \phi \frac{\partial}{\partial \theta}+\frac{1}{\mathrm{r}} \cos \theta \cos \phi \frac{\partial^{2}}{\partial \phi \partial \theta}\right]
$$

Expanding,

$$
\frac{1}{\mathrm{r}^{2}} \frac{\cos \theta \sin ^{2} \phi}{\sin \theta} \frac{\partial}{\partial \theta}-\frac{1}{\mathrm{r}^{2}} \frac{\cos \phi \cos \theta \sin \phi}{\sin \theta} \frac{\partial^{2}}{\partial \phi \partial \theta}
$$

Factoring,

$$
-\frac{1}{\mathrm{r}^{2}} \frac{\cos \theta \sin \phi}{\sin \theta}\left(-\sin \phi \frac{\partial}{\partial \theta}+\cos \phi \frac{\partial^{2}}{\partial \phi \partial \theta}\right)
$$

Third-term operating on the third term $\left(\right.$ for $\left.\frac{\partial}{\partial x}\left(\frac{\partial}{\partial x}\right)\right)$.

$$
\left(-\frac{1}{\mathrm{r}} \frac{\sin \phi}{\sin \theta} \frac{\partial}{\partial \phi}\right)\left(-\frac{1}{\mathrm{r}} \frac{\sin \phi}{\sin \theta} \frac{\partial}{\partial \phi}\right)
$$

The third term is a multiplication of functions:

$-\frac{1}{\mathrm{r}} \frac{\sin \phi}{\sin \theta}$

Function

(1)

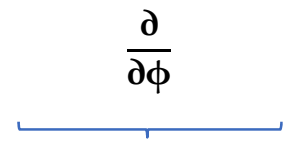

Function

(2)

$$
-\frac{1}{\mathrm{r}} \frac{\sin \phi}{\sin \theta} \frac{\partial}{\partial \phi}\left[-\frac{1}{\mathrm{r}} \frac{\sin \phi}{\sin \theta} \frac{\partial}{\partial \phi}\right]
$$

Performing just the derivative instruction:

$$
\frac{\partial}{\partial \phi}\left[-\frac{1}{r} \frac{\sin \phi}{\sin \theta} \frac{\partial}{\partial \phi}\right]=\left[-\frac{1}{r} \frac{\cos \phi}{\sin \theta} \frac{\partial}{\partial \phi}-\frac{1}{r} \frac{\sin \phi}{\sin \theta} \frac{\partial^{2}}{\partial \phi^{2}}\right]
$$


Inserting the result of our derivative in the original expression:

$$
-\frac{1}{\mathrm{r}} \frac{\sin \phi}{\sin \theta}\left[-\frac{1}{\mathrm{r}} \frac{\cos \phi}{\sin \theta} \frac{\partial}{\partial \phi}-\frac{1}{\mathrm{r}} \frac{\sin \phi}{\sin \theta} \frac{\partial^{2}}{\partial \phi^{2}}\right]
$$

Expanding,

$$
\frac{1}{\mathrm{r}^{2}} \frac{\cos \phi \sin \phi}{\sin ^{2} \theta} \frac{\partial}{\partial \phi}+\frac{1}{\mathrm{r}^{2}} \frac{\sin ^{2} \phi}{\sin ^{2} \theta} \frac{\partial^{2}}{\partial \phi^{2}}
$$

Factoring,

$$
\frac{1}{\mathrm{r}^{2}} \frac{\sin \phi}{\sin ^{2} \theta}\left(\cos \phi \frac{\partial}{\partial \phi}+\sin \phi \frac{\partial^{2}}{\partial \phi^{2}}\right)
$$

Section corresponding to $\frac{\partial}{\partial y}\left(\frac{\partial}{\partial y}\right)$.

$$
\begin{gathered}
\frac{\partial}{\partial y}\left(\frac{\partial}{\partial y}\right)=\left[\sin \theta \sin \phi \frac{\partial}{\partial r}+\frac{1}{r} \cos \theta \sin \phi \frac{\partial}{\partial \theta}+\frac{1}{r} \frac{\cos \phi}{\sin \theta} \frac{\partial}{\partial \phi}\right] \\
{\left[\sin \theta \sin \phi \frac{\partial}{\partial r}+\frac{1}{r} \cos \theta \sin \phi \frac{\partial}{\partial \theta}+\frac{1}{r} \frac{\cos \phi}{\sin \theta} \frac{\partial}{\partial \phi}\right]}
\end{gathered}
$$

Again, we have to operate term to term. Since we have three terms for each $\frac{\partial}{\partial y}$, we have nine terms at the end of this procedure section.

First-term operating on the first term $\left(\right.$ for $\left.\frac{\partial}{\partial y}\left(\frac{\partial}{\partial y}\right)\right)$.

$$
\left(\sin \theta \sin \phi \frac{\partial}{\partial \mathrm{r}}\right)\left(\sin \theta \sin \phi \frac{\partial}{\partial \mathrm{r}}\right)
$$

Both $\sin \theta$ and $\sin \phi$ are constant when operated by $\frac{\partial}{\partial r}$; thus,

$$
\sin ^{2} \theta \sin ^{2} \phi \frac{\partial^{2}}{\partial r^{2}}
$$

First-term operating on the second term $\left(\right.$ for $\left.\frac{\partial}{\partial y}\left(\frac{\partial}{\partial y}\right)\right)$.

$$
\left(\sin \theta \sin \phi \frac{\partial}{\partial \mathrm{r}}\right)\left(\frac{1}{\mathrm{r}} \cos \theta \sin \phi \frac{\partial}{\partial \theta}\right)
$$

We treat the second term as a multiplication of functions:

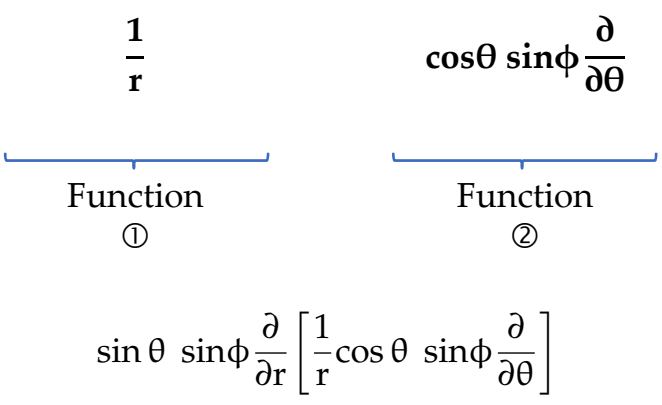

Performing just the derivative instruction:

$$
\frac{\partial}{\partial \mathrm{r}}\left[\frac{1}{\mathrm{r}} \cos \theta \sin \phi \frac{\partial}{\partial \theta}\right]=\left[\left(-\frac{1}{\mathrm{r}^{2}}\right) \cos \theta \sin \phi \frac{\partial}{\partial \theta}+\left(\frac{1}{\mathrm{r}}\right) \cos \theta \sin \phi \frac{\partial^{2}}{\partial \mathrm{r} \partial \theta}\right]
$$

Inserting the result of our derivative in the original expression:

$$
\sin \theta \sin \phi\left[\left(-\frac{1}{\mathrm{r}^{2}}\right) \cos \theta \sin \phi \frac{\partial}{\partial \theta}+\left(\frac{1}{\mathrm{r}}\right) \cos \theta \sin \phi \frac{\partial^{2}}{\partial \mathrm{r} \partial \theta}\right]
$$


Expanding,

$$
-\frac{1}{\mathrm{r}^{2}} \cos \theta \sin \theta \sin ^{2} \phi \frac{\partial}{\partial \theta}+\frac{1}{\mathrm{r}} \cos \theta \sin \theta \sin ^{2} \phi \frac{\partial^{2}}{\partial \mathrm{r} \partial \theta}
$$

Factoring,

$$
\frac{1}{\mathrm{r}^{2}} \cos \theta \sin \theta \sin ^{2} \phi\left(-\frac{\partial}{\partial \theta}+\mathrm{r} \frac{\partial^{2}}{\partial \mathrm{r} \partial \theta}\right)
$$

First-term operating on the third term $\left(\right.$ for $\left.\frac{\partial}{\partial y}\left(\frac{\partial}{\partial y}\right)\right)$.

$$
\left(\sin \theta \sin \phi \frac{\partial}{\partial \mathrm{r}}\right)\left(\frac{1}{\mathrm{r}} \frac{\cos \phi}{\sin \theta} \frac{\partial}{\partial \phi}\right)
$$

We treat the third term as a multiplication of functions:

$$
\begin{aligned}
& \frac{1}{\mathrm{r}} \quad \frac{\cos \phi}{\sin \theta} \frac{\partial}{\partial \phi} \\
& \text { Function } \\
& \text { (1) } \\
& \sin \theta \sin \phi \frac{\partial}{\partial \mathrm{r}}\left[\frac{1}{\mathrm{r}} \frac{\cos \phi}{\sin \theta} \frac{\partial}{\partial \phi}\right]
\end{aligned}
$$

Performing just the derivative instruction:

$$
\frac{\partial}{\partial \mathrm{r}}\left[\frac{1}{\mathrm{r}} \frac{\cos \phi}{\sin \theta} \frac{\partial}{\partial \phi}\right]=\left[\left(-\frac{1}{\mathrm{r}^{2}}\right) \frac{\cos \phi}{\sin \theta} \frac{\partial}{\partial \phi}+\left(\frac{1}{\mathrm{r}}\right) \frac{\cos \phi}{\sin \theta} \frac{\partial^{2}}{\partial \mathrm{r} \partial \phi}\right]
$$

Inserting the result of our derivative in the original expression:

$$
\sin \theta \sin \phi\left[-\frac{1}{\mathrm{r}^{2}} \frac{\cos \phi}{\sin \theta} \frac{\partial}{\partial \phi}+\frac{1}{\mathrm{r}} \frac{\cos \phi}{\sin \theta} \frac{\partial^{2}}{\partial \mathrm{r} \partial \phi}\right]
$$

Expanding,

$$
-\frac{1}{\mathrm{r}^{2}} \cos \phi \sin \phi \frac{\partial}{\partial \phi}+\frac{1}{\mathrm{r}} \cos \phi \sin \phi \frac{\partial^{2}}{\partial \mathrm{r} \partial \phi}
$$

Factoring,

$$
\frac{1}{\mathrm{r}^{2}} \cos \phi \sin \phi\left(-\frac{\partial}{\partial \phi}+r \frac{\partial^{2}}{\partial \mathrm{r} \partial \phi}\right)
$$

Second-term operating on the first term $\left(\right.$ for $\left.\frac{\partial}{\partial y}\left(\frac{\partial}{\partial y}\right)\right)$.

$$
\left(\frac{1}{\mathrm{r}} \cos \theta \sin \phi \frac{\partial}{\partial \theta}\right)\left(\sin \theta \sin \phi \frac{\partial}{\partial \mathrm{r}}\right)
$$

We treat the first term as a multiplication of functions:

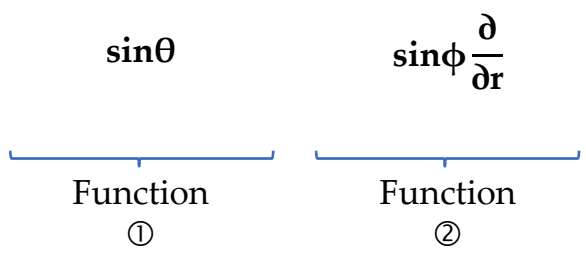




$$
\frac{1}{\mathrm{r}} \cos \theta \sin \phi \frac{\partial}{\partial \theta}\left[\sin \theta \sin \phi \frac{\partial}{\partial \mathrm{r}}\right]
$$

Performing just the derivative instruction:

$$
\frac{\partial}{\partial \theta}\left[\sin \theta \sin \phi \frac{\partial}{\partial r}\right]=\left[(\cos \theta) \sin \phi \frac{\partial}{\partial r}+(\sin \theta) \sin \phi \frac{\partial^{2}}{\partial \theta \partial r}\right]
$$

Inserting the result of our derivative in the original expression:

$$
\frac{1}{\mathrm{r}} \cos \theta \sin \phi\left[\cos \theta \sin \phi \frac{\partial}{\partial \mathrm{r}}+\sin \theta \sin \phi \frac{\partial^{2}}{\partial \theta \partial \mathrm{r}}\right]
$$

Expanding,

$$
\frac{1}{r} \cos ^{2} \theta \sin ^{2} \phi \frac{\partial}{\partial r}+\frac{1}{r} \cos \theta \sin \theta \sin ^{2} \phi \frac{\partial^{2}}{\partial \theta \partial r}
$$

Factoring,

$$
\frac{1}{\mathrm{r}} \cos \theta \sin ^{2} \phi\left(\cos \theta \frac{\partial}{\partial \mathrm{r}}+\sin \theta \frac{\partial^{2}}{\partial \theta \partial \mathrm{r}}\right)
$$

Second-term operating on the second term $\left(\right.$ for $\left.\frac{\partial}{\partial y}\left(\frac{\partial}{\partial y}\right)\right)$.

$$
\left(\frac{1}{r} \cos \theta \sin \phi \frac{\partial}{\partial \theta}\right)\left(\frac{1}{r} \cos \theta \sin \phi \frac{\partial}{\partial \theta}\right)
$$

We treat the second term as a multiplication of two functions:

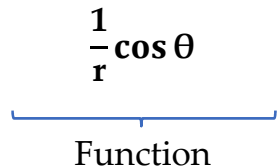

(1)

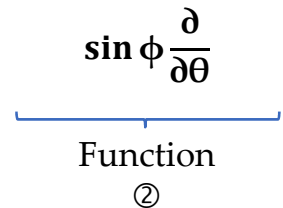

(2)

$$
\frac{1}{r} \cos \theta \sin \phi \frac{\partial}{\partial \theta}\left[\frac{1}{r} \cos \theta \sin \phi \frac{\partial}{\partial \theta}\right]
$$

Performing just the derivative instruction:

$$
\frac{\partial}{\partial \theta}\left[\frac{1}{r} \cos \theta \sin \phi \frac{\partial}{\partial \theta}\right]=\left[\left(-\frac{1}{r} \sin \theta\right) \sin \phi \frac{\partial}{\partial \theta}+\left(\frac{1}{r} \cos \theta\right) \sin \phi \frac{\partial^{2}}{\partial \theta^{2}}\right]
$$

Inserting the result of our derivative in the original expression:

$$
\frac{1}{r} \cos \theta \sin \phi\left[-\frac{1}{r} \sin \theta \sin \phi \frac{\partial}{\partial \theta}+\frac{1}{r} \cos \theta \sin \phi \frac{\partial^{2}}{\partial \theta^{2}}\right]
$$

Expanding,

$$
-\frac{1}{\mathrm{r}^{2}} \cos \theta \sin \theta \sin ^{2} \phi \frac{\partial}{\partial \theta}+\frac{1}{\mathrm{r}^{2}} \cos ^{2} \theta \sin ^{2} \phi \frac{\partial^{2}}{\partial \theta^{2}}
$$

Factoring,

$$
\frac{1}{\mathrm{r}^{2}} \cos \theta \sin ^{2} \phi\left(-\sin \theta \frac{\partial}{\partial \theta}+\cos \theta \frac{\partial^{2}}{\partial \theta^{2}}\right)
$$

Second-term operating on the third term $\left(\right.$ for $\left.\frac{\partial}{\partial y}\left(\frac{\partial}{\partial y}\right)\right)$.

$$
\left(\frac{1}{r} \cos \theta \sin \phi \frac{\partial}{\partial \theta}\right)\left(\frac{1}{r} \frac{\cos \phi}{\sin \theta} \frac{\partial}{\partial \phi}\right)
$$


We treat the second term as a multiplication of two functions:

$\frac{1}{\mathrm{r}} \frac{\cos \phi}{\sin \theta}$

Function

(1)

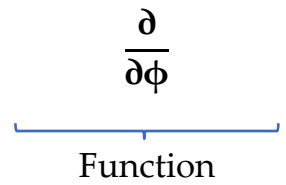

(2)

$$
\frac{1}{\mathrm{r}} \cos \theta \sin \phi \frac{\partial}{\partial \theta}\left[\frac{1}{\mathrm{r}} \frac{\cos \phi}{\sin \theta} \frac{\partial}{\partial \phi}\right]
$$

Performing just the derivative instruction:

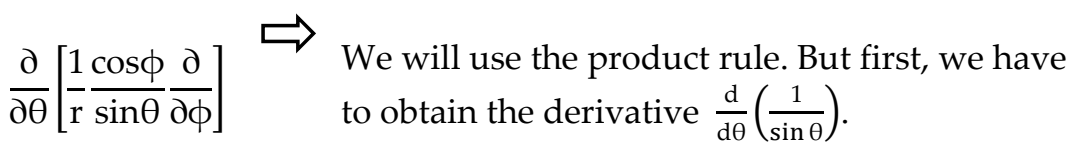

Makin a change of variable: $u=\sin \theta$, we have

$$
\begin{gathered}
\frac{d}{d u}\left(\frac{1}{u}\right) \frac{d u}{d \theta} \Rightarrow \frac{d}{d u}\left(\frac{1}{u}\right)=-\frac{1}{u^{2}} \\
\frac{d u}{d \theta}=\frac{d(\sin \theta)}{d \theta}=\cos \theta \\
\frac{d}{d \theta}\left(\frac{1}{\sin \theta}\right)=\frac{d}{d u}\left(\frac{1}{u}\right) \frac{d u}{d \theta}=-\frac{1}{u^{2}}(\cos \theta)=-\frac{1}{\sin ^{2} \theta} \cos \theta=-\frac{\cos \theta}{\sin ^{2} \theta}
\end{gathered}
$$

Going back to or derivative expression,

$$
\frac{\partial}{\partial \theta}\left[\frac{1}{r} \frac{\cos \phi}{\sin \theta} \frac{\partial}{\partial \phi}\right]=\left[-\frac{1}{r} \frac{\cos \theta \cos \phi}{\sin ^{2} \theta} \frac{\partial}{\partial \phi}+\frac{1}{r} \frac{\cos \phi}{\sin \theta} \frac{\partial^{2}}{\partial \theta \partial \phi}\right]
$$

Inserting the result of our derivative in the original expression:

$$
\frac{1}{\mathrm{r}} \cos \theta \sin \phi\left[-\frac{1}{\mathrm{r}} \frac{\cos \theta \cos \phi}{\sin ^{2} \theta} \frac{\partial}{\partial \phi}+\frac{1}{\mathrm{r}} \frac{\cos \phi}{\sin \theta} \frac{\partial^{2}}{\partial \theta \partial \phi}\right]
$$

Expanding,

$$
-\frac{1}{\mathrm{r}^{2}} \frac{\cos ^{2} \theta \cos \phi \sin \phi}{\sin ^{2} \theta} \frac{\partial}{\partial \phi}+\frac{1}{\mathrm{r}^{2}} \frac{\cos \theta \cos \phi \sin \phi}{\sin \theta} \frac{\partial^{2}}{\partial \theta \partial \phi}
$$

Factoring,

$$
-\frac{1}{\mathrm{r}^{2}} \frac{\cos \theta \cos \phi \operatorname{sen} \phi}{\sin \theta}\left(\frac{\cos \theta}{\sin \theta} \frac{\partial}{\partial \phi}-\frac{\partial^{2}}{\partial \theta \partial \phi}\right)
$$

Third-term operating on the first term $\left(\right.$ for $\left.\frac{\partial}{\partial y}\left(\frac{\partial}{\partial y}\right)\right)$.

$$
\left(\frac{1}{\mathrm{r}} \frac{\cos \phi}{\sin \theta} \frac{\partial}{\partial \phi}\right)\left(\sin \theta \sin \phi \frac{\partial}{\partial \mathrm{r}}\right)
$$

We treat the first term as multiplication of functions:

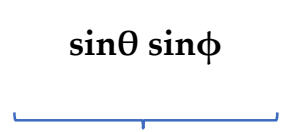

Function

(1) $\frac{\partial}{\partial r}$

Function

(2) 


$$
\frac{1}{\mathrm{r}} \frac{\cos \phi}{\sin \theta} \frac{\partial}{\partial \phi}\left[\sin \theta \sin \phi \frac{\partial}{\partial \mathrm{r}}\right]
$$

Performing just the derivative instruction:

$$
\frac{\partial}{\partial \phi}\left[\sin \theta \sin \phi \frac{\partial}{\partial r}\right]=\left[\sin \theta \cos \phi \frac{\partial}{\partial r}+\sin \theta \sin \phi \frac{\partial^{2}}{\partial \phi \partial r}\right]
$$

Inserting the result of our derivative in the original expression:

$$
\frac{1}{\mathrm{r}} \frac{\cos \phi}{\sin \theta}\left[\sin \theta \cos \phi \frac{\partial}{\partial \mathrm{r}}+\sin \theta \sin \phi \frac{\partial^{2}}{\partial \phi \partial \mathrm{r}}\right]
$$

Expanding,

$$
\frac{1}{\mathrm{r}} \cos ^{2} \phi \frac{\partial}{\partial \mathrm{r}}+\frac{1}{\mathrm{r}} \cos \phi \sin \phi \frac{\partial^{2}}{\partial \phi \partial \mathrm{r}}
$$

Factoring,

$$
\frac{1}{\mathrm{r}} \cos \phi\left(\cos \phi \frac{\partial}{\partial \mathrm{r}}+\sin \phi \frac{\partial^{2}}{\partial \phi \partial \mathrm{r}}\right)
$$

Third-term operating on the second term $\left(\right.$ for $\left.\frac{\partial}{\partial y}\left(\frac{\partial}{\partial y}\right)\right)$.

$$
\left(\frac{1}{\mathrm{r}} \frac{\cos \phi}{\sin \theta} \frac{\partial}{\partial \phi}\right)\left(\frac{1}{\mathrm{r}} \cos \theta \sin \phi \frac{\partial}{\partial \theta}\right)
$$

Let us treat the second term as a multiplication of two functions:

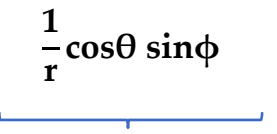

Function

(1)

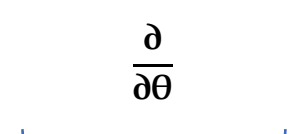

Function

(2)

$$
\frac{1}{\mathrm{r}} \frac{\cos \phi}{\sin \theta} \frac{\partial}{\partial \phi}\left[\frac{1}{\mathrm{r}} \cos \theta \sin \phi \frac{\partial}{\partial \theta}\right]
$$

Performing just the derivative instruction:

$$
\frac{\partial}{\partial \phi}\left[\frac{1}{\mathrm{r}} \cos \theta \sin \phi \frac{\partial}{\partial \theta}\right]=\left[\frac{1}{\mathrm{r}} \cos \theta \cos \phi \frac{\partial}{\partial \theta}+\frac{1}{\mathrm{r}} \cos \theta \sin \phi \frac{\partial^{2}}{\partial \phi \partial \theta}\right]
$$

Inserting the result of our derivative in the original expression:

$$
\frac{1}{\mathrm{r}} \frac{\cos \phi}{\sin \theta}\left[\frac{1}{\mathrm{r}} \cos \theta \cos \phi \frac{\partial}{\partial \theta}+\frac{1}{\mathrm{r}} \cos \theta \sin \phi \frac{\partial^{2}}{\partial \phi \partial \theta}\right]
$$

Expanding,

$$
\frac{1}{\mathrm{r}^{2}} \frac{\cos \theta \cos ^{2} \phi}{\sin \theta} \frac{\partial}{\partial \theta}+\frac{1}{\mathrm{r}^{2}} \frac{\cos \theta \cos \phi \sin \phi}{\sin \theta} \frac{\partial^{2}}{\partial \phi \partial \theta}
$$

Factoring,

$$
\frac{1}{\mathrm{r}^{2}} \frac{\cos \theta \cos \phi}{\sin \theta}\left(\cos \phi \frac{\partial}{\partial \theta}+\sin \phi \frac{\partial^{2}}{\partial \phi \partial \theta}\right)
$$

Third-term operating on the third term $\left(\right.$ for $\left.\frac{\partial}{\partial y}\left(\frac{\partial}{\partial y}\right)\right)$.

$$
\left(\frac{1}{\mathrm{r}} \frac{\cos \phi}{\sin \theta} \frac{\partial}{\partial \phi}\right)\left(\frac{1}{\mathrm{r}} \frac{\cos \phi}{\sin \theta} \frac{\partial}{\partial \phi}\right)
$$


Let us treat the third term as a multiplication of two functions:

$\frac{1}{\mathrm{r}} \frac{\cos \phi}{\sin \theta}$

Function

(1)

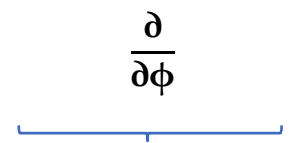

Function

(2)

$$
\frac{1}{\mathrm{r}} \frac{\cos \phi}{\sin \theta} \frac{\partial}{\partial \phi}\left[\frac{1}{\mathrm{r}} \frac{\cos \phi}{\sin \theta} \frac{\partial}{\partial \phi}\right]
$$

Performing just the derivative instruction:

$$
\frac{\partial}{\partial \phi}\left[\frac{1}{\mathrm{r}} \frac{\cos \phi}{\sin \theta} \frac{\partial}{\partial \phi}\right]=\left[-\frac{1}{\mathrm{r}} \frac{\sin \phi}{\sin \theta} \frac{\partial}{\partial \phi}+\frac{1}{\mathrm{r}} \frac{\cos \phi}{\sin \theta} \frac{\partial^{2}}{\partial \phi^{2}}\right]
$$

Inserting the result of our derivative in the original expression:

$$
\frac{1}{\mathrm{r}} \frac{\cos \phi}{\sin \theta}\left[-\frac{1}{\mathrm{r}} \frac{\sin \phi}{\sin \theta} \frac{\partial}{\partial \phi}+\frac{1}{\mathrm{r}} \frac{\cos \phi}{\sin \theta} \frac{\partial^{2}}{\partial \phi^{2}}\right]
$$

Expanding.

$$
-\frac{1}{\mathrm{r}^{2}} \frac{\cos \phi \sin \phi}{\sin ^{2} \theta} \frac{\partial}{\partial \phi}+\frac{1}{\mathrm{r}^{2}} \frac{\cos ^{2} \phi}{\sin ^{2} \theta} \frac{\partial^{2}}{\partial \phi^{2}}
$$

Factoring.

$$
\frac{1}{\mathrm{r}^{2}} \frac{\cos \phi}{\sin ^{2} \theta}\left(-\sin \phi \frac{\partial}{\partial \phi}+\cos \phi \frac{\partial^{2}}{\partial \phi^{2}}\right)
$$

Section corresponding to $\frac{\partial}{\partial z}\left(\frac{\partial}{\partial z}\right)$.

$$
\frac{\partial}{\partial z}\left(\frac{\partial}{\partial z}\right)=\left[\cos \theta \frac{\partial}{\partial r}-\frac{1}{r} \sin \theta \frac{\partial}{\partial \theta}\right]\left[\cos \theta \frac{\partial}{\partial r}-\frac{1}{r} \sin \theta \frac{\partial}{\partial \theta}\right]
$$

We have to operate term to term. Now, we only have a couple of terms for each $\frac{\partial}{\partial z}$. Therefore, we will have four terms at the end of this section of the procedure.

First-term operating on the first term $\left(\right.$ for $\left.\frac{\partial}{\partial z}\left(\frac{\partial}{\partial z}\right)\right)$.

$$
\left(\cos \theta \frac{\partial}{\partial \mathrm{r}}\right)\left(\cos \theta \frac{\partial}{\partial \mathrm{r}}\right)
$$

$\cos \theta$ is a constant function when operated by $\frac{\partial}{\partial \mathrm{r}}$. Therefore,

$$
\cos ^{2} \theta \frac{\partial^{2}}{\partial r^{2}}
$$

First-term operating on the second term $\left(\right.$ for $\left.\frac{\partial}{\partial z}\left(\frac{\partial}{\partial z}\right)\right)$.

$$
\left(\cos \theta \frac{\partial}{\partial r}\right)\left(-\frac{1}{r} \sin \theta \frac{\partial}{\partial \theta}\right)
$$


Let us treat the second term as a multiplication of two functions:

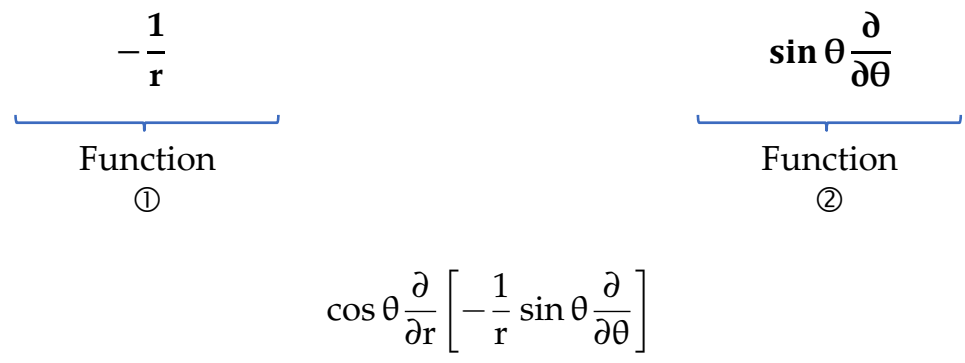

Performing just the derivative instruction:

$$
\frac{\partial}{\partial \mathrm{r}}\left[-\frac{1}{\mathrm{r}} \sin \theta \frac{\partial}{\partial \theta}\right]=\left[\left(\frac{1}{\mathrm{r}^{2}}\right) \sin \theta \frac{\partial}{\partial \theta}-\frac{1}{\mathrm{r}} \sin \theta \frac{\partial^{2}}{\partial \mathrm{r} \partial \theta}\right]
$$

Inserting the result of our derivative in the original expression:

$$
\cos \theta\left[\frac{1}{\mathrm{r}^{2}} \sin \theta \frac{\partial}{\partial \theta}-\frac{1}{\mathrm{r}} \sin \theta \frac{\partial^{2}}{\partial \mathrm{r} \partial \theta}\right]
$$

Expanding,

$$
\frac{1}{\mathrm{r}^{2}} \cos \theta \sin \theta \frac{\partial}{\partial \theta}-\frac{1}{\mathrm{r}} \cos \theta \sin \theta \frac{\partial^{2}}{\partial \mathrm{r} \partial \theta}
$$

Factoring,

$$
-\frac{1}{\mathrm{r}^{2}} \cos \theta \sin \theta\left(-\frac{\partial}{\partial \theta}+\mathrm{r} \frac{\partial^{2}}{\partial \mathrm{r} \partial \theta}\right)
$$

Second-term operating on the first term (para $\frac{\partial}{\partial z}\left(\frac{\partial}{\partial z}\right)$ ).

$$
\left(-\frac{1}{\mathrm{r}} \sin \theta \frac{\partial}{\partial \theta}\right)\left(\cos \theta \frac{\partial}{\partial \mathrm{r}}\right)
$$

Let us treat the first term as a multiplication of two functions:

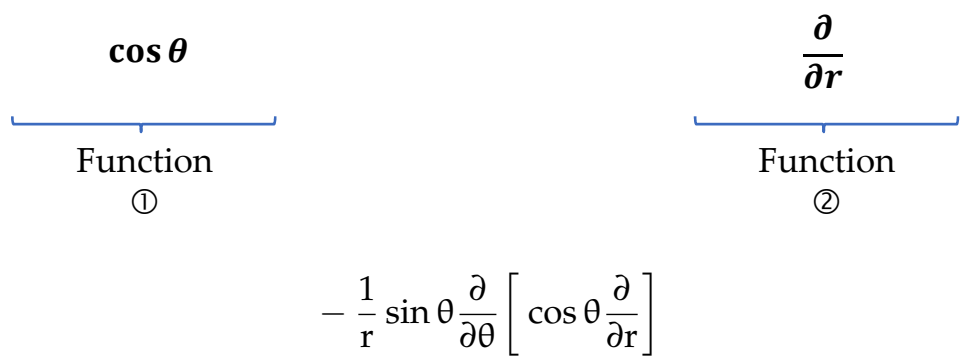

Performing just the derivative instruction:

$$
\frac{\partial}{\partial \theta}\left[\cos \theta \frac{\partial}{\partial r}\right]=\left[-\sin \theta \frac{\partial}{\partial r}+\cos \theta \frac{\partial^{2}}{\partial \theta \partial r}\right]
$$

Inserting the result of our derivative in the original expression:

$$
-\frac{1}{r} \sin \theta\left[-\sin \theta \frac{\partial}{\partial r}+\cos \theta \frac{\partial^{2}}{\partial \theta \partial r}\right]
$$

Expanding,

$$
\frac{1}{\mathrm{r}} \sin ^{2} \theta \frac{\partial}{\partial \mathrm{r}}-\frac{1}{\mathrm{r}} \cos \theta \sin \theta \frac{\partial^{2}}{\partial \theta \partial \mathrm{r}}
$$


Factoring,

$$
\frac{1}{\mathrm{r}} \sin \theta\left(\sin \theta \frac{\partial}{\partial \mathrm{r}}-\cos \theta \frac{\partial^{2}}{\partial \theta \partial \mathrm{r}}\right)
$$

Second-term operating on the second term $\left(\right.$ for $\left.\frac{\partial}{\partial z}\left(\frac{\partial}{\partial z}\right)\right)$.

$$
\left(-\frac{1}{\mathrm{r}} \sin \theta \frac{\partial}{\partial \theta}\right)\left(-\frac{1}{\mathrm{r}} \sin \theta \frac{\partial}{\partial \theta}\right)
$$

Let us treat the second term as a multiplication of two functions:

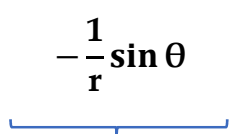

Function

(1)

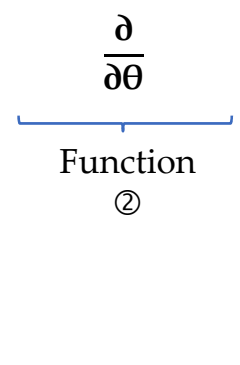

Performing just the derivative instruction:

$$
\frac{\partial}{\partial \theta}\left[-\frac{1}{\mathrm{r}} \sin \theta \frac{\partial}{\partial \theta}\right]=\left[-\frac{1}{\mathrm{r}} \cos \theta \frac{\partial}{\partial \theta}-\frac{1}{\mathrm{r}} \sin \theta \frac{\partial^{2}}{\partial \theta^{2}}\right]
$$

Inserting the result of our derivative in the original expression:

$$
-\frac{1}{\mathrm{r}} \sin \theta\left[-\frac{1}{\mathrm{r}} \cos \theta \frac{\partial}{\partial \theta}-\frac{1}{\mathrm{r}} \sin \theta \frac{\partial^{2}}{\partial \theta^{2}}\right]
$$

Expanding,

$$
\frac{1}{\mathrm{r}^{2}} \cos \theta \sin \theta \frac{\partial}{\partial \theta}+\frac{1}{\mathrm{r}^{2}} \sin ^{2} \theta \frac{\partial^{2}}{\partial \theta^{2}}
$$

Factoring,

$$
\frac{1}{\mathrm{r}^{2}} \sin \theta\left(\cos \theta \frac{\partial}{\partial \theta}+\sin \theta \frac{\partial^{2}}{\partial \theta^{2}}\right)
$$

At this moment, we have 22 terms, 9 of $\frac{\partial}{\partial x}\left(\frac{\partial}{\partial x}\right), 9$ of $\frac{\partial}{\partial y}\left(\frac{\partial}{\partial y}\right)$ and 4 of $\frac{\partial}{\partial z}\left(\frac{\partial}{\partial z}\right)$.

As we already discussed, the Laplacian in spherical coordinates is nothing more than adding those 22 expressions, that is to say,

$$
\nabla^{2}=\frac{\partial}{\partial x}\left(\frac{\partial}{\partial x}\right)+\frac{\partial}{\partial y}\left(\frac{\partial}{\partial y}\right)+\frac{\partial}{\partial z}\left(\frac{\partial}{\partial z}\right)
$$

We organize our terms using a convention of colors and lines as presented in Figure 4.

Sum of the underlined terms.

$$
\sin ^{2} \theta \cos ^{2} \phi \frac{\partial^{2}}{\partial \mathrm{r}^{2}}+\sin ^{2} \theta \sin ^{2} \phi \frac{\partial^{2}}{\partial \mathrm{r}^{2}}+\cos ^{2} \theta \frac{\partial^{2}}{\partial \mathrm{r}^{2}}
$$

Factoring,

$$
\frac{\partial^{2}}{\partial \mathrm{r}^{2}}\left[\sin ^{2} \theta \cos ^{2} \phi+\sin ^{2} \theta \sin ^{2} \phi+\cos ^{2} \theta\right]
$$


The expression in brackets has power 2 trigonometric functions. Therefore, we can use the following identities:

$$
\begin{aligned}
& \sin ^{2} \mathrm{~A}=\frac{1}{2}-\frac{1}{2} \cos (2 \mathrm{~A}) \\
& \cos ^{2} \mathrm{~A}=\frac{1}{2}+\frac{1}{2} \cos (2 \mathrm{~A})
\end{aligned}
$$

Let us apply these identities only to the first two terms enclosed in brackets.

$$
\begin{aligned}
& \frac{\partial^{2}}{\partial \mathrm{r}^{2}}\left(\left[\left(\frac{1}{2}-\frac{1}{2} \cos (2 \theta)\right)\left(\frac{1}{2}+\frac{1}{2} \cos (2 \phi)\right)\right]+\left[\left(\frac{1}{2}-\frac{1}{2} \cos (2 \theta)\right)\left(\frac{1}{2}-\frac{1}{2} \cos (2 \phi)\right)\right]+\left[\cos ^{2} \theta\right]\right) \\
& \frac{\partial^{2}}{\partial \mathrm{r}^{2}}\left\{\left[\frac{1}{4}-\frac{1}{4} \cos (2 \theta)+\frac{1}{4} \cos (2 \phi)-\frac{1}{4} \cos (2 \theta) \cos (2 \phi)\right]+\left[\frac{1}{4}-\frac{1}{4} \cos (2 \theta)-\frac{1}{4} \cos (2 \phi)+\frac{1}{4} \cos (2 \theta) \cos (2 \phi)\right]+\left[\cos ^{2} \theta\right]\right\} \\
& \frac{\partial^{2}}{\partial \mathrm{r}^{2}}\left[\left(\frac{1}{2}-\frac{1}{2} \cos (2 \theta)\right)+\cos ^{2} \theta\right] \\
& \frac{\partial^{2}}{\partial \mathrm{r}^{2}}\left[\operatorname{sen}^{2} \theta+\cos ^{2} \theta\right]=\frac{\partial^{2}}{\partial \mathrm{r}^{2}} \\
& -\frac{1}{\mathrm{r}} \cos \theta \sin \theta \cos ^{2} \phi\left(\frac{\partial}{\partial \theta}-\mathrm{r} \frac{\partial^{2}}{\partial \mathrm{r} \partial \theta}\right)+\frac{1}{\mathrm{r}^{2}} \cos \theta \sin \theta \sin ^{2} \phi\left(-\frac{\partial}{\partial \theta}+\mathrm{r} \frac{\partial^{2}}{\partial \mathrm{r} \partial \theta}\right)-\frac{1}{\mathrm{r}^{2}} \cos \theta \sin \theta\left(-\frac{\partial}{\partial \theta}+\mathrm{r} \frac{\partial^{2}}{\partial \mathrm{r} \partial \theta}\right)
\end{aligned}
$$

We can factor the operators in parentheses as follows:

$$
\left(\frac{1}{\mathrm{r}^{2}}\right)(\cos \theta \sin \theta)\left(-1+\cos ^{2} \phi+\sin ^{2} \phi\right)\left(-\frac{\partial}{\partial \theta}+\mathrm{r} \frac{\partial^{2}}{\partial \mathrm{r} \partial \theta}\right)
$$

We expand the following multiplication:

$$
\begin{gathered}
(\cos \theta \sin \theta)\left(-1+\cos ^{2} \phi+\sin ^{2} \phi\right) \\
=\left[-\cos \theta \sin \theta+\cos \theta \sin \theta \cos ^{2} \phi+\cos \theta \sin \theta \sin ^{2} \phi\right]
\end{gathered}
$$

Again, we use the trigonometric identities of power 2 to rewrite the previous expression:

$$
-\cos \theta \sin \theta+\cos \theta \sin \theta\left(\frac{1}{2}+\frac{1}{2} \cos (2 \phi)\right)+\cos \theta \sin \theta\left(\frac{1}{2}-\frac{1}{2} \cos (2 \phi)\right)
$$

Expanding:

$-\cos \theta \sin \theta+\frac{1}{2} \cos \theta \sin \theta+\frac{1}{2} \cos \theta \sin \theta \cos (2 \phi)+\frac{1}{2} \cos \theta \sin \theta-\frac{1}{2} \cos \theta \sin \theta \cos (2 \phi)$

$$
=-\cos \theta \sin \theta+\frac{1}{2} \cos \theta \sin \theta+\frac{1}{2} \cos \theta \sin \theta=0
$$

Therefore, we have

$$
\begin{gathered}
\left(\frac{1}{\mathrm{r}^{2}}\right)(\cos \theta \sin \theta)\left(-1+\cos ^{2} \phi+\sin ^{2} \phi\right)\left(-\frac{\partial}{\partial \theta}+\mathrm{r} \frac{\partial^{2}}{\partial \mathrm{r} \partial \theta}\right)=0 \\
\left(\frac{1}{\mathrm{r}^{2}}\right)(0)\left(-\frac{\partial}{\partial \theta}+\mathrm{r} \frac{\partial^{2}}{\partial \mathrm{r} \partial \theta}\right)=0
\end{gathered}
$$




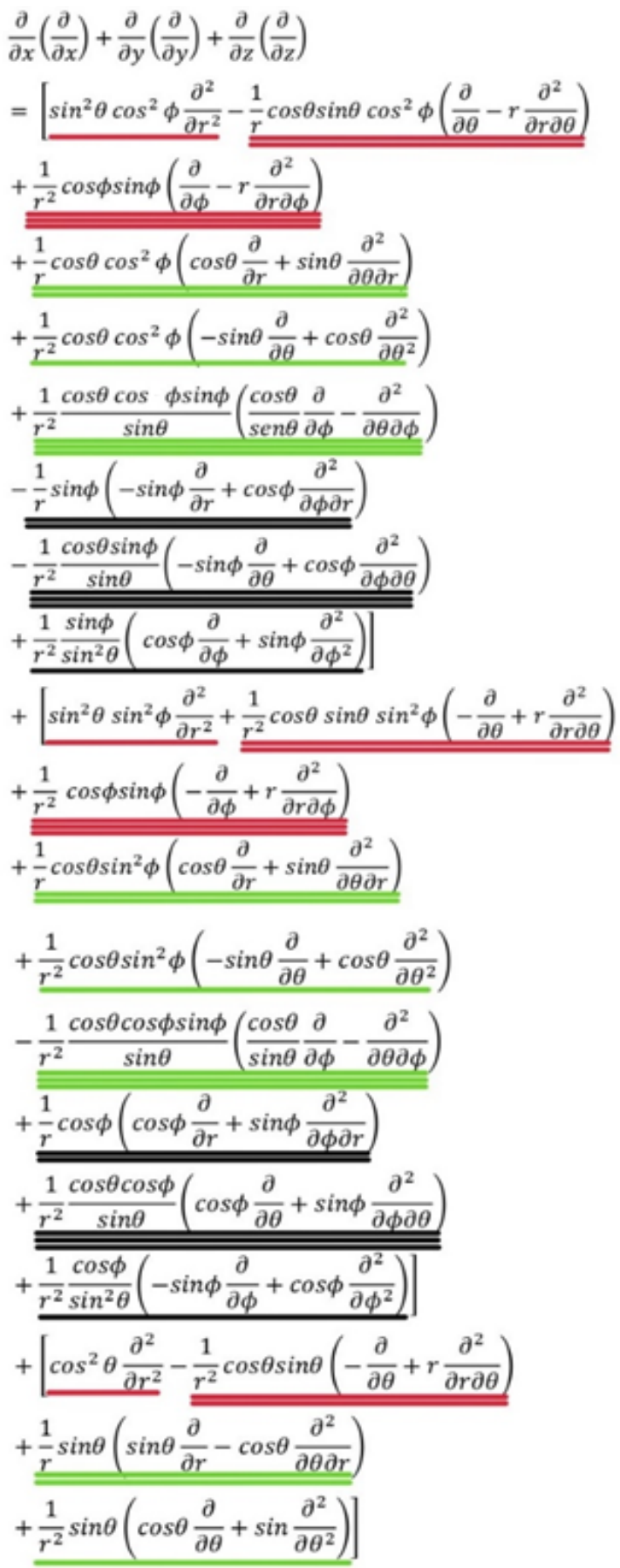

Figure 4. The sum of all the terms shown in this figure will be the Laplacian operator in spherical polar coordinates. To organize the sums conveniently, we will be guided by the terms that contain second derivatives. Terms that contain $\frac{\partial^{2}}{\partial r^{2}}$ are underlined with a single red line. Terms containing $\frac{\partial^{2}}{\partial r \partial \theta}$ are underlined with a double red line. Terms containing $\frac{\partial^{2}}{\partial r \partial \phi}$ are underlined with a triple red line. Terms that contain $\frac{\partial^{2}}{\partial \theta^{2}}$ are underlined with a single green line. Terms containing $\frac{\partial^{2}}{\partial \theta \partial r}$ are underlined with a double green line. Terms containing $\frac{\partial^{2}}{\partial \theta \partial \phi}$ are underlined with a triple green line. Terms that contain $\frac{\partial^{2}}{\partial \phi^{2}}$ are underlined with a single black line. Terms containing $\frac{\partial^{2}}{\partial \phi \partial r}$ are underlined with a double black line. Terms containing $\frac{\partial^{2}}{\partial \phi \partial \theta}$ are underlined with a triple black line. 
Sum of the underlined terms.

$$
\frac{1}{\mathrm{r}^{2}} \cos \phi \sin \phi\left(\frac{\partial}{\partial \phi}-\mathrm{r} \frac{\partial^{2}}{\partial \mathrm{r} \partial \phi}\right)+\frac{1}{\mathrm{r}^{2}} \cos \phi \sin \phi\left(-\frac{\partial}{\partial \phi}+\mathrm{r} \frac{\partial^{2}}{\partial \mathrm{r} \partial \phi}\right)
$$

One term is positive and the other is negative; therefore, they cancel, $=0$.

Sum of the underlined terms.

$$
\frac{1}{\mathrm{r}^{2}} \cos \theta \cos ^{2} \phi\left(-\sin \theta \frac{\partial}{\partial \theta}+\cos \theta \frac{\partial^{2}}{\partial \theta^{2}}\right)+\frac{1}{\mathrm{r}^{2}} \cos \theta \sin ^{2} \phi\left(-\sin \theta \frac{\partial}{\partial \theta}+\cos \theta \frac{\partial^{2}}{\partial \theta^{2}}\right)+\frac{1}{\mathrm{r}^{2}} \sin \theta\left(\cos \theta \frac{\partial}{\partial \theta}+\sin \theta \frac{\partial^{2}}{\partial \theta^{2}}\right)
$$

In this case, we should expand the terms and organize them as follows:

$$
\frac{1}{\mathrm{r}^{2}} \cos \theta \sin \theta \frac{\partial}{\partial \theta}-\frac{1}{\mathrm{r}^{2}} \cos \theta \sin \theta \cos ^{2} \phi \frac{\partial}{\partial \theta}-\frac{1}{\mathrm{r}^{2}} \cos \theta \sin \theta \operatorname{sen}^{2} \phi \frac{\partial}{\partial \theta}+\frac{1}{\mathrm{r}^{2}} \cos ^{2} \theta \cos ^{2} \phi \frac{\partial^{2}}{\partial \theta^{2}}+\frac{1}{\mathrm{r}^{2}} \cos ^{2} \theta \sin ^{2} \phi \frac{\partial^{2}}{\partial \theta^{2}}+\frac{1}{\mathrm{r}^{2}} \sin ^{2} \theta \frac{\partial^{2}}{\partial \theta^{2}}
$$

The first three terms that are multiplying $\frac{\partial}{\partial \theta}$ can be factored as follows:

$$
\left(-\frac{1}{\mathrm{r}^{2}}\right)(\cos \theta \sin \theta)\left(-1+\cos ^{2} \phi+\sin ^{2} \phi\right)\left(\frac{\partial}{\partial \theta}\right)
$$

Let us recall that $\cos ^{2} \phi+\sin ^{2} \phi=1$; then,

$$
\left(-\frac{1}{\mathrm{r}^{2}}\right)(\cos \theta \sin \theta)(-1+1)\left(\frac{\partial}{\partial \theta}\right)=\left(-\frac{1}{\mathrm{r}^{2}}\right)(\cos \theta \sin \theta)(0)\left(\frac{\partial}{\partial \theta}\right)=0
$$
as follows:

In the case of the second three terms that are multiplying $\frac{\partial^{2}}{\partial \theta^{2}}$, they can be factored

$$
\left(\frac{1}{r^{2}}\right)\left(\frac{\partial^{2}}{\partial \theta^{2}}\right)\left(\cos ^{2} \theta \cos ^{2} \phi+\cos ^{2} \theta \sin ^{2} \phi+\sin ^{2} \theta\right)
$$

We use the trigonometric identities of power 2 to rewrite the previous expression

$$
\left.\left(\frac{1}{\mathrm{r}^{2}}\right)\left(\frac{\partial^{2}}{\partial \theta^{2}}\right)\left\{\left(\frac{1}{2}+\frac{1}{2} \cos (2 \theta)\right)\left(\frac{1}{2}+\frac{1}{2} \cos (2 \phi)\right)\right]+\left[\left(\frac{1}{2}+\frac{1}{2} \cos (2 \theta)\right)\left(\frac{1}{2}-\frac{1}{2} \cos (2 \phi)\right)\right]+\left[\sin ^{2} \theta\right]\right\}
$$

Expanding:

$$
\left(\frac{1}{r^{2}}\right)\left(\frac{\partial^{2}}{\partial \theta^{2}}\right)\left\{\left[\frac{1}{4}+\frac{1}{4} \cos (2 \theta)+\frac{1}{4} \cos (2 \phi)+\frac{1}{4} \cos (2 \theta) \cos (2 \phi)\right]+\left[\frac{1}{4}+\frac{1}{4} \cos (2 \theta)-\frac{1}{4} \cos (2 \phi)-\frac{1}{4} \cos (2 \theta) \cos (2 \phi)\right]+\left[\sin ^{2} \theta\right]\right\}
$$

We simplify:

$$
\left(\frac{1}{\mathrm{r}^{2}}\right)\left(\frac{\partial^{2}}{\partial \theta^{2}}\right)\left[\left(\frac{1}{2}+\frac{1}{2} \cos (2 \theta)\right)+\sin ^{2} \theta\right]
$$

Let us recall that $\frac{1}{2}+\frac{1}{2} \cos (2 \theta)=\cos ^{2} \theta$, and $\cos ^{2} \phi+\sin ^{2} \phi=1$; then

$$
\left(\frac{1}{\mathrm{r}^{2}}\right)\left(\frac{\partial^{2}}{\partial \theta^{2}}\right)\left(\cos ^{2} \theta+\sin ^{2} \theta\right)=\left(\frac{1}{\mathrm{r}^{2}}\right)\left(\frac{\partial^{2}}{\partial \theta^{2}}\right)
$$

Sum of the underlined terms.

$$
\begin{gathered}
\frac{1}{\mathrm{r}} \cos \theta \cos ^{2} \phi\left(\cos \theta \frac{\partial}{\partial \mathrm{r}}+\sin \theta \frac{\partial^{2}}{\partial \theta \partial \mathrm{r}}\right)+\frac{1}{\mathrm{r}} \cos \theta \sin ^{2} \phi\left(\cos \theta \frac{\partial}{\partial \mathrm{r}}+\sin \theta \frac{\partial^{2}}{\partial \theta \partial \mathrm{r}}\right) \\
+\frac{1}{\mathrm{r}} \sin \theta\left(\sin \theta \frac{\partial}{\partial \mathrm{r}}-\cos \theta \frac{\partial^{2}}{\partial \theta \partial \mathrm{r}}\right)
\end{gathered}
$$


Again, we should expand the terms and accommodate them as follows:

$$
\begin{gathered}
\frac{1}{\mathrm{r}} \cos ^{2} \theta \cos ^{2} \phi \frac{\partial}{\partial \mathrm{r}}+\frac{1}{\mathrm{r}} \cos ^{2} \theta \sin ^{2} \phi \frac{\partial}{\partial \mathrm{r}}+\frac{1}{\mathrm{r}} \sin ^{2} \theta \frac{\partial}{\partial \mathrm{r}} \\
+\frac{1}{\mathrm{r}} \cos \theta \sin \theta \cos ^{2} \phi \frac{\partial^{2}}{\partial \theta \partial \mathrm{r}}+\frac{1}{\mathrm{r}} \cos \theta \sin \theta \sin ^{2} \phi \frac{\partial^{2}}{\partial \theta \partial \mathrm{r}}-\frac{1}{\mathrm{r}} \cos \theta \sin \theta \frac{\partial^{2}}{\partial \theta \partial \mathrm{r}}
\end{gathered}
$$

The first three terms that multiply $\frac{\partial}{\partial \mathrm{r}}$ can be factored as follows:

$$
\frac{1}{\mathrm{r}} \frac{\partial}{\partial \mathrm{r}}\left[\cos ^{2} \theta \cos ^{2} \phi+\cos ^{2} \theta \sin ^{2} \phi+\sin ^{2} \theta\right]
$$

We already reviewed; therefore, we are sure that the term

$$
\cos ^{2} \theta \cos ^{2} \phi+\cos ^{2} \theta \sin ^{2} \phi+\sin ^{2} \theta=1
$$

So, for the first three terms, we have

$$
\frac{1}{\mathrm{r}} \frac{\partial}{\partial \mathrm{r}}
$$

For the case of the second three terms that multiply $\frac{\partial^{2}}{\partial \theta \partial \mathrm{r}}$, they can be factored as follows:

$$
\left(\frac{1}{r}\right)\left((\cos \theta \sin \theta)\left(-1+\cos ^{2} \phi+\sin ^{2} \phi\right)\right)\left(\frac{\partial^{2}}{\partial \theta \partial r}\right)
$$

We already checked; therefore, we are sure that the multiplication

$$
(\cos \theta \sin \theta)\left(-1+\cos ^{2} \phi+\sin ^{2} \phi\right)=0
$$

Therefore, the sum of the terms that multiply $\frac{\partial^{2}}{\partial \theta \partial \mathrm{r}}$ equals zero and the only term that survives from the sum for is

$$
\frac{1}{\mathrm{r}} \frac{\partial}{\partial \mathrm{r}}
$$

Sum of the underlined terms

$\frac{1}{\mathrm{r}^{2}} \frac{\cos \theta \cos ^{2} \phi \sin \phi}{\sin \theta}\left(\frac{\cos \theta}{\sin \theta} \frac{\partial}{\partial \phi}-\frac{\partial^{2}}{\partial \theta \partial \phi}\right)-\frac{1}{\mathrm{r}^{2}} \frac{\cos \theta \cos \phi \sin \phi}{\sin \theta}\left(\frac{\cos \theta}{\sin \theta} \frac{\partial}{\partial \phi}-\frac{\partial^{2}}{\partial \theta \partial \phi}\right)$

One term is positive and the other is negative. Therefore, they are canceled, $=0$.

Sum of the underlined terms.

$$
\frac{1}{\mathrm{r}^{2}} \frac{\sin \phi}{\sin ^{2} \theta}\left(\cos \phi \frac{\partial}{\partial \phi}+\sin \phi \frac{\partial^{2}}{\partial \phi^{2}}\right)+\frac{1}{\mathrm{r}^{2}} \frac{\cos \phi}{\sin ^{2} \theta}\left(-\sin \phi \frac{\partial}{\partial \phi}+\cos \phi \frac{\partial^{2}}{\partial \phi^{2}}\right)
$$

Expanding, we have

$$
\begin{aligned}
\frac{1}{\mathrm{r}^{2}} \frac{\sin \phi \cos \phi}{\sin ^{2} \theta} \frac{\partial}{\partial \phi} & +\frac{1}{\mathrm{r}^{2}} \frac{\sin ^{2} \phi}{\sin ^{2} \theta} \frac{\partial^{2}}{\partial \phi^{2}}-\frac{1}{\mathrm{r}^{2}} \frac{\sin \phi \cos \phi}{\sin ^{2} \theta} \frac{\partial}{\partial \phi}+\frac{1}{\mathrm{r}^{2}} \frac{\cos ^{2} \phi}{\sin ^{2} \theta} \frac{\partial^{2}}{\partial \phi^{2}} \\
& =\frac{1}{\mathrm{r}^{2}} \frac{\sin ^{2} \phi}{\sin ^{2} \theta} \frac{\partial^{2}}{\partial \phi^{2}}+\frac{1}{\mathrm{r}^{2}} \frac{\cos ^{2} \phi}{\sin ^{2} \theta} \frac{\partial^{2}}{\partial \phi^{2}}
\end{aligned}
$$

We can factor the previous expression in the following way:

$$
\left[\frac{1}{\mathrm{r}^{2}} \frac{\left(\cos ^{2} \phi+\sin ^{2} \phi\right)}{\sin ^{2} \theta}\right]\left[\frac{\partial^{2}}{\partial \phi^{2}}\right]=\frac{1}{\mathrm{r}^{2} \sin ^{2} \theta} \frac{\partial^{2}}{\partial \phi^{2}}
$$


Sum of the underlined terms.

$$
-\frac{1}{\mathrm{r}} \sin \phi\left(-\sin \phi \frac{\partial}{\partial \mathrm{r}}+\cos \phi \frac{\partial^{2}}{\partial \phi \partial \mathrm{r}}\right)+\frac{1}{\mathrm{r}} \cos \phi\left(\cos \phi \frac{\partial}{\partial \mathrm{r}}+\sin \phi \frac{\partial^{2}}{\partial \phi \partial \mathrm{r}}\right)
$$

Expanding, we have

$$
\begin{gathered}
\frac{1}{\mathrm{r}} \sin ^{2} \phi \frac{\partial}{\partial \mathrm{r}}-\frac{1}{\mathrm{r}} \sin \phi \cos \phi \frac{\partial^{2}}{\partial \phi \partial \mathrm{r}}+\frac{1}{\mathrm{r}} \cos ^{2} \phi \frac{\partial}{\partial \mathrm{r}}+\frac{1}{\mathrm{r}} \sin \phi \cos \phi \frac{\partial^{2}}{\partial \phi \partial \mathrm{r}} \\
=\frac{1}{\mathrm{r}} \sin ^{2} \phi \frac{\partial}{\partial \mathrm{r}}+\frac{1}{\mathrm{r}} \cos ^{2} \phi \frac{\partial}{\partial r}
\end{gathered}
$$

Factoring,

$$
\frac{1}{\mathrm{r}}\left(\sin ^{2} \phi+\cos ^{2} \phi\right) \frac{\partial}{\partial \mathrm{r}}=\frac{1}{\mathrm{r}} \frac{\partial}{\partial \mathrm{r}}
$$

Sum of the underlined terms.

$$
-\frac{1}{\mathrm{r}^{2}} \frac{\cos \theta \sin \phi}{\sin \theta}\left(-\sin \phi \frac{\partial}{\partial \theta}+\cos \phi \frac{\partial^{2}}{\partial \phi \partial \theta}\right)+\frac{1}{\mathrm{r}^{2}} \frac{\cos \theta \cos \phi}{\sin \theta}\left(\cos \phi \frac{\partial}{\partial \theta}+\sin \phi \frac{\partial^{2}}{\partial \phi \partial \theta}\right)
$$

Expanding, we have

$$
\begin{gathered}
\frac{1}{\mathrm{r}^{2}} \frac{\cos \theta \sin ^{2} \phi}{\sin \theta} \frac{\partial}{\partial \theta}-\frac{1}{\mathrm{r}^{2}} \frac{\cos \theta \cos \phi \sin \phi}{\sin \theta} \frac{\partial^{2}}{\partial \phi \partial \theta}+\frac{1}{\mathrm{r}^{2}} \frac{\cos \theta \cos ^{2} \phi}{\sin \theta} \frac{\partial}{\partial \theta}+\frac{1}{\mathrm{r}^{2}} \frac{\cos \theta \cos \phi \sin \phi}{\sin \theta} \frac{\partial^{2}}{\partial \phi \partial \theta} \\
=\frac{1}{\mathrm{r}^{2}} \frac{\cos \theta \sin ^{2} \phi}{\sin \theta} \frac{\partial}{\partial \theta}+\frac{1}{\mathrm{r}^{2}} \frac{\cos \theta \cos ^{2} \phi}{\sin \theta} \frac{\partial}{\partial \theta}
\end{gathered}
$$

Factoring,

$$
\left(\frac{1}{\mathrm{r}^{2}}\right)\left(\frac{\cos \theta}{\sin \theta}\right)\left(\sin ^{2} \phi+\cos ^{2} \phi\right)\left(\frac{\partial}{\partial \theta}\right)=\frac{1}{\mathrm{r}^{2}} \frac{\cos \theta}{\sin \theta} \frac{\partial}{\partial \theta}
$$

Now, we can write the Laplacian, adding the results of the sums that we carried out.

$$
\begin{gathered}
\frac{\partial}{\partial x}\left(\frac{\partial}{\partial x}\right)+\frac{\partial}{\partial y}\left(\frac{\partial}{\partial y}\right)+\frac{\partial}{\partial z}\left(\frac{\partial}{\partial z}\right)=\frac{\partial^{2}}{\partial r^{2}}+0+0+\frac{1}{r^{2}} \frac{\partial^{2}}{\partial \phi^{2}}+\frac{1}{r} \frac{\partial}{\partial r}+0 \\
+\frac{1}{r^{2} \sin ^{2} \theta} \frac{\partial^{2}}{\partial \phi^{2}}+\frac{1}{r} \frac{\partial}{\partial r}+\frac{1}{r^{2}} \frac{\cos \theta}{\sin \theta} \frac{\partial}{\partial \theta}
\end{gathered}
$$

Simplifying,

$$
\nabla^{2}=\frac{\partial^{2}}{\partial r^{2}}+\frac{2}{r} \frac{\partial}{\partial r}+\frac{1}{r^{2}} \frac{\cos \theta}{\sin \theta} \frac{\partial}{\partial \theta}+\frac{1}{r^{2}} \frac{\partial^{2}}{\partial \theta^{2}}+\frac{1}{r^{2} \sin ^{2} \theta} \frac{\partial^{2}}{\partial \phi^{2}}
$$

Finally, let us identify that the Laplacian in spherical coordinates that appears in the literature is "slightly different" from the one obtained here and that we present in Equation (5). Actually, there is no difference; the only thing that happens is that the operators for $r$ and for $\theta$ are nested in order to only present three terms in the Laplacian. Let us briefly review this. 
The Laplacian that commonly appears in the literature is

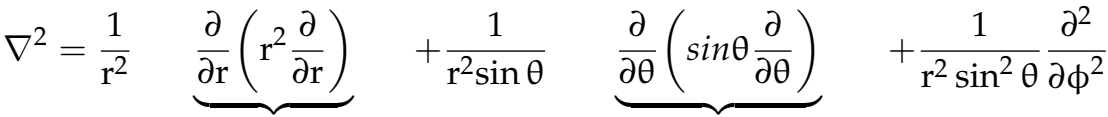

$$
\begin{aligned}
& \text { In this case, the } \\
& \text { operator } \frac{\partial}{\partial r} \\
& \text { operates on a } \\
& \text { multiplication } \\
& \text { of } 2 \text { functions, } \\
& r^{2} \text { and } \frac{\partial}{\partial r}
\end{aligned}
$$

When expanding the operators, we have

$$
\begin{gathered}
\frac{1}{\mathrm{r}^{2}} \frac{\partial}{\partial \mathrm{r}}\left(\mathrm{r}^{2} \frac{\partial}{\partial \mathrm{r}}\right) \\
\frac{\partial}{\partial \mathrm{r}}\left[\mathrm{r}^{2} \frac{\partial}{\partial \mathrm{r}}\right]=\left[2 \mathrm{r} \frac{\partial}{\partial \mathrm{r}}+\mathrm{r}^{2} \frac{\partial^{2}}{\partial \mathrm{r}^{2}}\right]
\end{gathered}
$$

so that

$$
\begin{array}{r}
\frac{1}{\mathrm{r}^{2}}\left[2 \mathrm{r} \frac{\partial}{\partial \mathrm{r}}+\mathrm{r}^{2} \frac{\partial^{2}}{\partial \mathrm{r}^{2}}\right]=\underbrace{}_{\begin{array}{c}
\text { These two are } \\
\text { the first terms } \\
\text { of Equation (5) }
\end{array}} \frac{2 \frac{\partial}{\partial r}+\frac{\partial^{2}}{\partial r^{2}}}{\partial{ }^{2}} \\
\frac{1}{\mathrm{r}^{2} \sin \theta} \frac{\partial}{\partial \theta}\left(\sin \theta \frac{\partial}{\partial \theta}\right) \\
\frac{\partial}{\partial \theta}\left[\sin \theta \frac{\partial}{\partial \theta}\right]=\left[\cos \theta \frac{\partial}{\partial \theta}+\sin \theta \frac{\partial^{2}}{\partial \theta^{2}}\right]
\end{array}
$$

Therefore, we have

$$
\frac{1}{\mathrm{r}^{2} \sin \theta}\left[\cos \theta \frac{\partial}{\partial \theta}+\sin \theta \frac{\partial^{2}}{\partial \theta^{2}}\right]=\underbrace{\frac{\cos \theta}{\mathrm{r}^{2} \sin \theta} \frac{\partial}{\partial \theta}+\frac{1}{\mathrm{r}^{2}} \frac{\partial^{2}}{\partial \theta^{2}}}_{\begin{array}{c}
\text { These two are the terms } 3 \\
\text { and } 4 \text { of Equation }(5) \\
\text { corresponding to the } \\
\text { operator of } \theta
\end{array}}
$$

With the above, we corroborate the equivalence between both expressions. Therefore, we can also write the Laplacian in spherical coordinates as follows:

$$
\nabla^{2}=\frac{1}{r^{2}} \frac{\partial}{\partial r}\left(r^{2} \frac{\partial}{\partial r}\right)+\frac{1}{r^{2} \sin \theta} \frac{\partial}{\partial \theta}\left(\sin \theta \frac{\partial}{\partial \theta}\right)+\frac{1}{r^{2} \sin ^{2} \theta} \frac{\partial^{2}}{\partial \phi}
$$

\section{Conclusions}

In this paper, we present a full-fledged derivation of the Laplacian operator in spherical coordinates. The mathematical concepts that we used for this purpose are available to all STEM disciplines beginning in the second year of undergraduate. Therefore, this solution is accessible for physics and science students and engineering and chemistry students. Our referral procedure is completely self-contained; this allows the reader to work self-taught with our document without resorting to a complimentary bibliography. This work can be useful for students and academics whose studies and research make use of this important mathematical tool. 
Author Contributions: Conceptualization, A.R.-G.; Funding acquisition, A.R.-G.; Methodology, A.R.-G.; Supervision, A.R.-G.; Writing-original draft, A.L.P.-M., M.d.P.A.-D.-V. and A.R.-G. All authors have read and agreed to the published version of the manuscript.

Funding: This research received no external funding.

Institutional Review Board Statement: Not applicable.

Informed Consent Statement: Not applicable.

Acknowledgments: The authors are deeply grateful for the excellent peer review this paper received. We also acknowledge and appreciate the invaluable labor and courage of all those who, during the tragic COVID-19 pandemic, expose their lives to keep doing first necessity activities for all society's good. This research work was financially supported by the following projects: (a) PAPIIT-UNAM, project number IA107218 under the technical administration of AL Pérez-Martínez; (b) Investigación Científica Básica SEP-CONACYT project number 283492; (c) PAPIIT-UNAM, project number: IN109020 under the technical administration of A. Rodríguez-Gómez. All authors declare their faith and gratitude to almighty God.

Conflicts of Interest: The authors declare no conflict of interest.

\section{References}

1. Batchelor, G.K. An Introduction to Fluid Dynamics; Cambridge University Press: Cambridge, UK, 2000; ISBN 9780521663960.

2. Purcell, E. Electricity and Magnetism, 2nd ed.; Cambridge University Press: Cambridge, UK, 2011; ISBN 9781139005043.

3. Griffiths, D.J. Introduction to Electrodynamics, 4th ed.; Pearson Education, I., Ed.; Cambridge University Press: Cambridge, UK, 2017; ISBN 9781108420419.

4. Thornton, S.T.; Rex, A. Modern Physics for Scientists and Engineers, 4th ed.; Dodd, E., Ed.; Cengage Learning: Boston, MA, USA, 2013; ISBN 9781133103721.

5. Beiser, A. Concepts of Modern Physics, 6th ed.; McGraw-Hill Higher Education: New York, NY, USA, 2003; ISBN 007115096X.

6. Tipler, P.A.; Llewellyn, R.A. Modern Physics, 5th ed.; W. H. Freeman and Company: New York, NY, USA, 2008; ISBN 9780716775508.

7. Serway, R.A.; Moses, C.J.; Moyer, C.A. Modern Physiscs, 3rd ed.; Thomson Learning: Belmont, CA, USA, $2005 ;$ ISBN 0534493394.

8. Taylor, P.L.; Heinonen, O. A Quantum Approach to Condensed Matter Physics; Cambridge University Press: New York, NY, USA, 2002; ISBN 052177103X.

9. Grøn, Ø.; Næss, A. Einstein's Theory; Springer: New York, NY, USA, 2011; ISBN 9781461407058.

10. Brehm, J.J.; Mullin, W.J. Introduction to the Structure of Matter: A Course in Modern Physics; John Wiley \& Sons, Inc.: Hoboken, NJ, USA, 1989; ISBN 9780471605317.

11. Shankar, R. Principles of Quantum Mechanics, 2nd ed.; Plenum Publishers; Kluwer Academic: New York, NY, USA, 1994.

12. Liboff, R.L. Introductory Quantum Mechanics; Longman Higher Education: Boston, MA, USA, 1987; ISBN 0201122219.

13. Cohen-Tannoudji, C.; Diu, B.; Laloe, F. Quantum Mechanics; Wiley-Interscience: New York, NY, USA, 1991 ; ISBN 0471569526.

14. Dirac, P.A.M. The Principles of Quantum Mechanics, 4th ed.; Oxford University Press: Oxford, UK, 1958 ; ISBN 0198520115.

15. Schwartz, A.T. The Periodic Table: Its Story and Its Significance (Eric R. Scerri). J. Chem. Educ. 2007, 84, 598. [CrossRef]

16. Scerri, E. The Periodic Table: Its Story and Its Significance; Oxford University Press: Oxford, UK, $2019 ;$ ISBN 9780190914363.

17. Koch, W.; Frenking, G.; Gauss, J.; Cremer, D.; Collins, J.R. Helium chemistry: Theoretical predictions and experimental challenge. J. Am. Chem. Soc. 1987, 109, 5917-5934. [CrossRef]

18. Hoeper, P.S.; Wenstrup, R.S. Numerical Solution to Laplace's Equation in Spherical Coordinates with Axial Symmetry. J. Appl. Phys. 1970, 41, 1879-1882. [CrossRef]

19. DiStasio, M.; McHarris, W.C. Electrostatic problems? Relax! Am. J. Phys. 1979, 47, 440-444. [CrossRef]

20. LI, S.; Funaki, H. Discrete Spherical Laplacian Operator. IEICE Trans. Inf. Syst. 2016, E99.D, 1716-1719. [CrossRef]

21. Feng, J.Q.; Beard, K.V. A Perturbation Model of Raindrop Oscillation Characteristics with Aerodynamic Effects. J. Atmos. Sci. 1991, 48, 1856-1868. [CrossRef]

22. Maurits, N.; Ćurčić-Blake, B. Math for Scientists, 1st ed.; Springer International Publishing: Cham, Switzerland, 2017; ISBN 9783319573533.

23. Boas, M.L. Mathematical Methods in The Physical Sciences, 3rd ed.; Wiley: Hoboken, NJ, USA, 2005; ISBN 9780471198260.

24. Chen, F.M. Laplacian Operator in Spherical Coordinates, an Alternative Derivation. Am. J. Phys. 1972, 40, 553-556. [CrossRef]

25. Katriel, J. A physically motivated derivation of the Laplacian in terms of the total angular momentum operator. Eur. J. Phys. 2019, 40, 045401. [CrossRef] 